\title{
Novel and Expeditious Microwave-Assisted Three-Component Reactions for the Synthesis of Spiroimidazolin-4-ones
}

\author{
Ping Ye, Katie Sargent, Ethan Stewart, Ji-Feng Liu, ${ }^{*}$ Daniel Yohannes, and Libing Yu \\ ArQule, Inc., 19 Presidential Way, Woburn, MA 10801, USA \\ jliu@arqule.com; iifeng.liu@yahoo.com
}

\section{Table of Contents}

General and Characterization Data....................................... 2 - 8

NMR spectra:

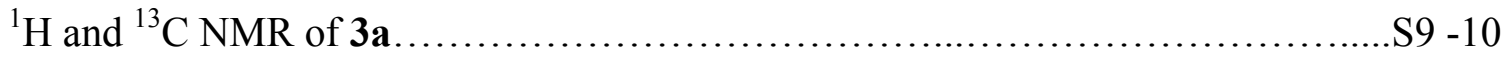

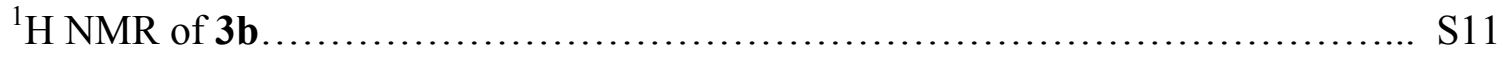

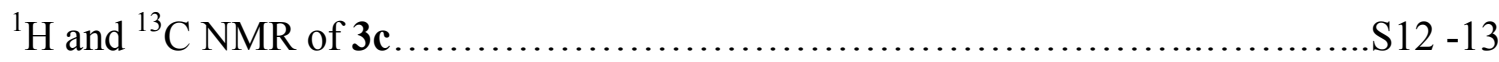

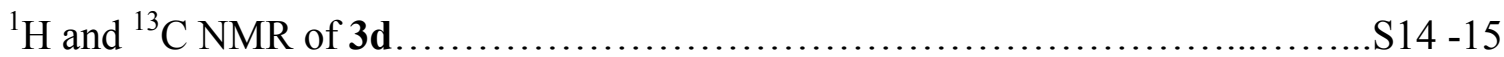

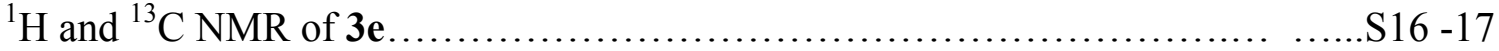

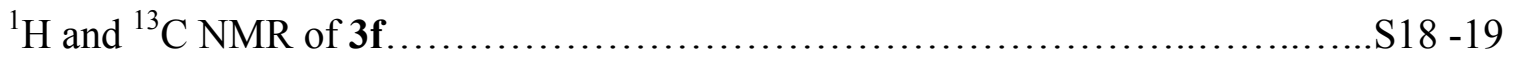

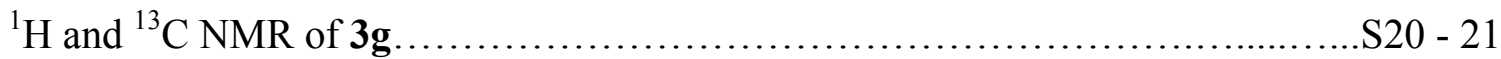

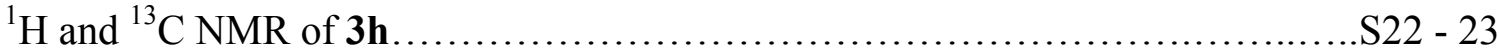

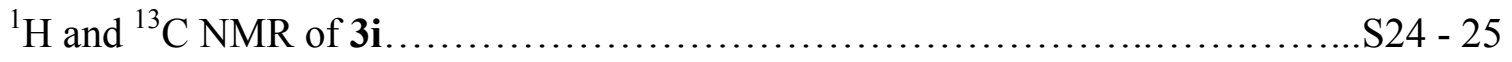

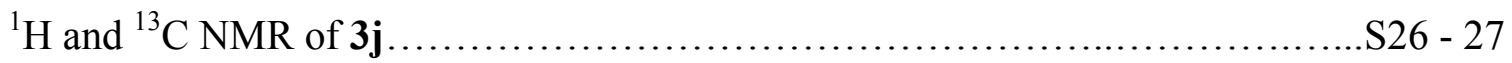

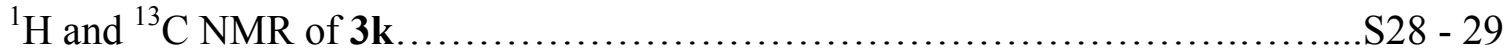

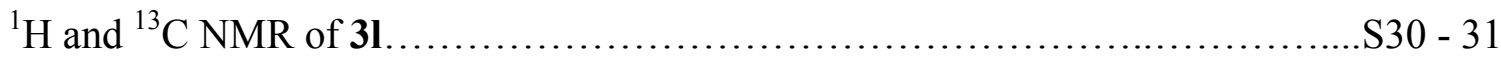

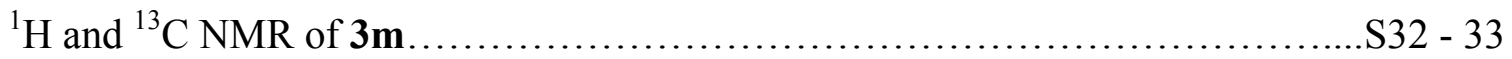

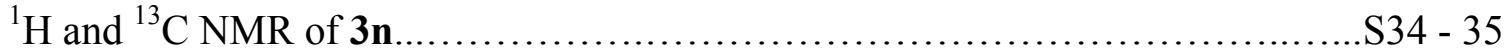

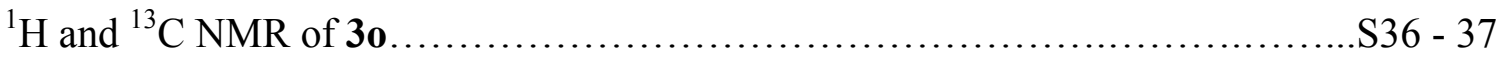

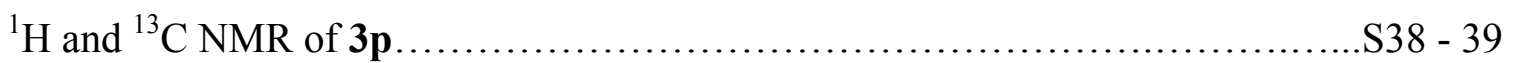

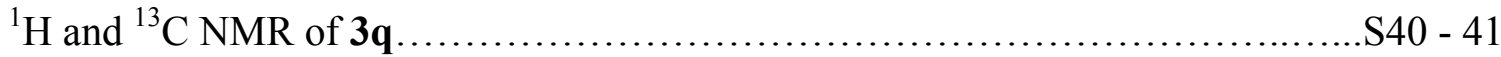

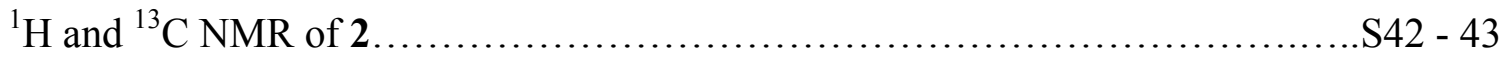




\section{SUPPORTING INFORMATION}

General. All reagents and solvents were purchased and used without further purification. Biotage Smith Synthesizer ${ }^{\mathrm{TM}}$, which were fully integrated with ArQule's proprietary Automated Molecular Assembly Plant (AMAP ${ }^{\mathrm{TM}}$ ), were used for the microwave reactions. Irradiation was initiated at $300 \mathrm{~W}$ to raise the temperature to the set point and then power was applied at intervals and levels to maintain the desired temperature. Reactions were run in sealed vessels. Pressure was between 8 to 15 Bar. Reaction times reported included time for the vial to ramp to the desired temperature. The product isolation was performed on silica gel $60 \mathrm{~F}_{254}$ prep-TLC plates. The Preparative HPLC was carried out employing a MACCEL 120-10-C18 column (50x20 $\mathrm{mm}$ ). The flow rate was at $88 \mathrm{~mL} / \mathrm{min}$ utilizing an acetonitrile/water mobile phase and $0.1 \%$ trifluoroacetic acid as a modifier. ${ }^{1} \mathrm{H}$ NMR $(300$, or $400 \mathrm{MHz})$ and ${ }^{13} \mathrm{C} \mathrm{NMR}(75$, or $100 \mathrm{MHz}$ ) spectra were recorded in $\mathrm{CDCl}_{3}$ or $\mathrm{DMSO}-\mathrm{d}_{6}$. Chemical shifts are given in parts per million ( $\mathrm{ppm}$ ) downfield from tetramethylsilane (TMS) as an internal reference, and coupling constants ( $J$-values) are in hertz $(\mathrm{Hz})$. Selected data are reported in the following manner: chemical shift; multiplicity $(\mathrm{s}=$ singlet, $\mathrm{d}=$ doublet, $\mathrm{t}=$ triplet, $\mathrm{m}=$ multiplet, $\mathrm{b}=$ broad); coupling constants and assignment. Low-resolution mass spectra were recorded in the $\mathrm{ES}^{+}$mode. The high-resolution mass spectra (HRMS) were recorded in the FAB mode.

\section{Characterization data:}

3-Benzyl-2-butyl-1,3-diaza-spiro[4.4]non-1-en-4-one (3a). Product was purified by prep-TLC (ethyl acetate/hexane $=1 / 1)$ as light yellow oil $(43 \mathrm{mg}, 75 \%) ;{ }^{1} \mathrm{H}$ NMR (400 $\left.\mathrm{MHz}, \mathrm{CDCl}_{3}\right) \delta 0.85(\mathrm{t}, J=7.4 \mathrm{~Hz}, 3 \mathrm{H}), 1.26-1.35(\mathrm{~m}, 2 \mathrm{H}), 1.51-1.58(\mathrm{~m}, 2 \mathrm{H}), 1.80-$ $1.83(\mathrm{~m}, 2 \mathrm{H}), 1.94-2.02(\mathrm{~m}, 6 \mathrm{H}), 2.29(\mathrm{t}, J=7.8 \mathrm{~Hz}, 2 \mathrm{H}), 4.68(\mathrm{~s}, 2 \mathrm{H}), 7.13-7.16(\mathrm{~m}$, 2H), $7.26-7.36(\mathrm{~m}, 3 \mathrm{H}) ;{ }^{13} \mathrm{C} \mathrm{NMR}\left(75 \mathrm{MHz}, \mathrm{CDCl}_{3}\right) \delta 13.6,22.3,26.1,27.7,28.8,37.4$, 43.5, 76.5, 126.7, 127.7, 128.9, 136.6, 161.7, 186.7; MS m/z 285 (M+H); HRMS calcd. for $\left(\mathrm{C}_{18} \mathrm{H}_{24} \mathrm{~N}_{2} \mathrm{O}+\mathrm{H}\right) 285.1961$, found 285.1968 . 


\section{SUPPORTING INFORMATION}

2-Benzyl-3-(2-methoxy-ethyl)-1,3-diaza-spiro[4.4]non-1-en-4-one (3b). Product was purified by prep-TLC (ethyl acetate/hexane $=1 / 1)$ as light yellow oil $(43 \mathrm{mg}, 75 \%) ;{ }^{1} \mathrm{H}$ NMR $\left(300 \mathrm{MHz}, \mathrm{CDCl}_{3}\right) \delta 1.83-2.05(\mathrm{~m}, 8 \mathrm{H}), 3.29(\mathrm{~s}, 3 \mathrm{H}), 3.33(\mathrm{t}, J=5.4 \mathrm{~Hz}, 2 \mathrm{H})$, $3.44(\mathrm{t}, J=5.4 \mathrm{~Hz}, 2 \mathrm{H}), 3.98(\mathrm{~s}, 2 \mathrm{H}), 7.25-7.36(\mathrm{~m}, 5 \mathrm{H}) ; \mathrm{MS} m / z 287(\mathrm{M}+\mathrm{H})$; HRMS calcd. for $\left(\mathrm{C}_{17} \mathrm{H}_{22} \mathrm{~N}_{2} \mathrm{O}_{2}+\mathrm{H}\right)$ 287.1754, found 287.1762 .

2-Benzyl-3-(2-methoxy-ethyl)-1,3-diaza-spiro[4.5]dec-1-en-4-one (3c). Product was purified by prep-TLC (ethyl acetate/hexane $=1 / 1)$ as light yellow oil $(39 \mathrm{mg}, 66 \%) ;{ }^{1} \mathrm{H}$ NMR (400 MHz, $\left.\mathrm{CDCl}_{3}\right) . \delta 1.48-1.52(\mathrm{~m}, 2 \mathrm{H}), 1.71-1.86(\mathrm{~m}, 8 \mathrm{H}), 3.29(\mathrm{~s}, 3 \mathrm{H}), 3.32$ $(\mathrm{t}, J=5.4 \mathrm{~Hz}, 2 \mathrm{H}), 3.42(\mathrm{t}, J=5.4 \mathrm{~Hz}, 2 \mathrm{H}), 4.00(\mathrm{~s}, 2 \mathrm{H}), 7.24-7.35(\mathrm{~m}, 5 \mathrm{H}) ;{ }^{13} \mathrm{C} \mathrm{NMR}$ $\left(100 \mathrm{MHz}, \mathrm{CDCl}_{3}\right) \delta 21.5,25.2,33.2,35.8,40.4,59.1,70.0,70.3,127.2,128.4,128.9$, 134.6, 160.7, 186.4; MS m/z $301(\mathrm{M}+\mathrm{H})$; HRMS calcd. for $\left(\mathrm{C}_{18} \mathrm{H}_{24} \mathrm{~N}_{2} \mathrm{O}_{2}+\mathrm{H}\right)$ 301.1910, found 301.1913 .

2-Benzyl-3-(2-methoxy-ethyl)-5,5-dimethyl-3,5-dihydro-imidazol-4-one (3d). Product was purified by prep-TLC (ethyl acetate/hexane =1/1) as light yellow oil (34 mg, 65\%); ${ }^{1} \mathrm{H}$ NMR (400 MHz, $\left.\mathrm{CDCl}_{3}\right) \delta 1.49(\mathrm{~s}, 6 \mathrm{H}), 3.33(\mathrm{~s}, 3 \mathrm{H}), 3.41(\mathrm{t}, J=5.2 \mathrm{~Hz}, 2 \mathrm{H}), 3.56(\mathrm{t}$, $J=5.2 \mathrm{~Hz}, 2 \mathrm{H}), 4.21(\mathrm{~s}, 2 \mathrm{H}), 7.27-7.39(\mathrm{~m}, 5 \mathrm{H}) ;{ }^{13} \mathrm{C} \mathrm{NMR}\left(100 \mathrm{MHz}, \mathrm{CDCl}_{3}\right) \delta 23.9$, $35.8,40.6,59.1,67.1,70.3,127.3,128.5,129.0,134.4,160.9,186.5 ; \mathrm{MS} m / z 261(\mathrm{M}+\mathrm{H})$; HRMS calcd. for $\left(\mathrm{C}_{15} \mathrm{H}_{20} \mathrm{~N}_{2} \mathrm{O}_{2}+\mathrm{H}\right) 261.1598$, found 261.1606 .

2,5-Dibenzyl-3-(2-methoxy-ethyl)-5-methyl-3,5-dihydro-imidazol-4-one (3e). Product was purified by prep-TLC (ethyl acetate/hexane $=1 / 1$ ) as a light yellow solid (50 mg, $75 \%) ;{ }^{1} \mathrm{H}$ NMR (400 MHz, $\left.\mathrm{CDCl}_{3}\right) \delta 1.46(\mathrm{~s}, 3 \mathrm{H}), 2.79-2.84(\mathrm{~m}, 1 \mathrm{H}), 3.07(\mathrm{~d}, J=13.2$ $\mathrm{Hz}, 1 \mathrm{H}), 3.15(\mathrm{~d}, J=13.2 \mathrm{~Hz}, 1 \mathrm{H}), 3.10-3.27(\mathrm{~m}, 3 \mathrm{H}), 3.11(\mathrm{~s}, 3 \mathrm{H}), 3.80(\mathrm{~s}, 2 \mathrm{H}), 6.84-$ $6.86(\mathrm{~m}, 2 \mathrm{H}), 7.15-7.23(\mathrm{~m}, 8 \mathrm{H}) ;{ }^{13} \mathrm{C} \mathrm{NMR}\left(100 \mathrm{MHz}, \mathrm{CDCl}_{3}\right) \delta$ 23.7, 35.7, 40.4, 43.5, 


\section{SUPPORTING INFORMATION}

$58.8,70.0,71.4,126.6,126.9,127.9,128.5,128.8,130.3,134.2,135.9,161.3,185.2 ; \mathrm{MS}$ $m / z 337(\mathrm{M}+\mathrm{H})$; HRMS calcd. for $\left(\mathrm{C}_{21} \mathrm{H}_{24} \mathrm{~N}_{2} \mathrm{O}_{2}+\mathrm{H}\right) 337.1910$, found 337.1918.

3-Benzyl-2-isobutyl-1,3-diaza-spiro[4.4]non-1-en-4-one (3f). Product was purified by prep-TLC (ethyl acetate/hexane $=1 / 1)$ as light yellow oil $(42 \mathrm{mg}, 74 \%) ;{ }^{1} \mathrm{H}$ NMR (400 $\left.\mathrm{MHz}, \mathrm{CDCl}_{3}\right) \delta 0.91(\mathrm{~d}, J=6.4 \mathrm{~Hz}, 6 \mathrm{H}), 1.81-1.84(\mathrm{~m}, 3 \mathrm{H}), 1.95-2.05(\mathrm{~m}, 6 \mathrm{H}), 2.17$ $(\mathrm{d}, J=6.8 \mathrm{~Hz}, 2 \mathrm{H}), 4.68(\mathrm{~s}, 2 \mathrm{H}), 7.14(\mathrm{~d}, J=7.2 \mathrm{~Hz}, 2 \mathrm{H}), 7.26-7.36(\mathrm{~m}, 3 \mathrm{H}) ;{ }^{13} \mathrm{C}$ NMR $\left(75 \mathrm{MHz}, \mathrm{CDCl}_{3}\right) \delta 22.3,26.0,26.1,37.5,37.7,43.6,76.6,126.6,127.7,128.9,136.5$, 160.9, 186.8; MS m/z $285(\mathrm{M}+\mathrm{H})$; HRMS calcd. for $\left(\mathrm{C}_{18} \mathrm{H}_{24} \mathrm{~N}_{2} \mathrm{O}+\mathrm{H}\right)$ 285.1961, found 285.1970 .

3-Benzyl-2-p-tolyl-1,3-diaza-spiro[4.4]non-1-en-4-one (3g). Product was purified by prep-TLC (ethyl acetate/hexane $=1 / 1)$ as a yellow solid $(43 \mathrm{mg}, 67 \%) ;{ }^{1} \mathrm{H}$ NMR (400 $\left.\mathrm{MHz} \mathrm{CDCl}_{3}\right) \delta 1.92-2.12(\mathrm{~m}, 8 \mathrm{H}), 2.37(\mathrm{~s}, 3 \mathrm{H}), 4.71(\mathrm{~s}, 2 \mathrm{H}), 7.01(\mathrm{dd}, J=7.8,1.8 \mathrm{~Hz}$ $2 \mathrm{H}), 7.16-7.18(\mathrm{~d}, J=8 \mathrm{~Hz}, 2 \mathrm{H}), 7.23-7.32(\mathrm{~m}, 5 \mathrm{H}) ;{ }^{13} \mathrm{C} \mathrm{NMR}\left(100 \mathrm{MHz}, \mathrm{CDCl}_{3}\right) \delta$ $21.4,26.1,37.7,44.8,77.1,126.7,126.9,127.5,128.1,128.7,129.2,136.7,140.9,161.0$, 187.2; MS m/z $319(\mathrm{M}+\mathrm{H})$; HRMS calcd. for $\left(\mathrm{C}_{21} \mathrm{H}_{22} \mathrm{~N}_{2} \mathrm{O}+\mathrm{H}\right) 319.1805$, found 319.1807. 2,3-Dibenzyl-1,3-diaza-spiro[4.4]non-1-en-4-one (3h). Product was purified by prepTLC (ethyl acetate/hexane $=1 / 1)$ as a light yellow solid $(41 \mathrm{mg}, 65 \%) ;{ }^{1} \mathrm{H}$ NMR (400 $\left.\mathrm{MHz}, \mathrm{CDCl}_{3}\right) \delta 1.88-2.12(\mathrm{~m}, 8 \mathrm{H}), 3.65(\mathrm{~s}, 2 \mathrm{H}), 4.43(\mathrm{~s}, 2 \mathrm{H}), 7.05-7.07(\mathrm{~m}, 2 \mathrm{H}), 7.17$ - $7.19(\mathrm{~m}, 2 \mathrm{H}), 7.26-7.35(\mathrm{~m}, 6 \mathrm{H}) ;{ }^{13} \mathrm{C} \mathrm{NMR}\left(100 \mathrm{MHz}, \mathrm{CDCl}_{3}\right) \delta$ 26.0, 36.1, 37.5, $43.3,77.0,126.5,127.4,127.7,128.2,128.9,129.0,134.0,136.4,160.0,186.5 ; \mathrm{MS} m / z$ $319(\mathrm{M}+\mathrm{H})$; HRMS calcd. for $\left(\mathrm{C}_{21} \mathrm{H}_{22} \mathrm{~N}_{2} \mathrm{O}+\mathrm{H}\right) 319.1805$, found 319.1812 .

3-Benzyl-2-pyridin-3-ylmethyl-1,3-diaza-spiro[4.4]non-1-en-4-one (3i). Product was purified by prep-TLC (ethyl acetate/hexane $=1 / 1)$ as a yellow solid $(37 \mathrm{mg}, 59 \%) ;{ }^{1} \mathrm{H}$ 


\section{SUPPORTING INFORMATION}

NMR $\left(400 \mathrm{MHz}, \mathrm{CDCl}_{3}\right) \delta 1.87-2.10(\mathrm{~m}, 8 \mathrm{H}), 3.65(\mathrm{~s}, 2 \mathrm{H}), 4.51(\mathrm{~s}, 2 \mathrm{H}), 7.05(\mathrm{dd}, J=$ 7.0, $1.0 \mathrm{~Hz}, 2 \mathrm{H}), 7.21-7.34(\mathrm{~m}, 4 \mathrm{H}), 7.48(\mathrm{~d}, J=8.0 \mathrm{~Hz}, 1 \mathrm{H}), 8.41(\mathrm{~d}, J=2.0 \mathrm{~Hz}, 1 \mathrm{H})$, $8.51(\mathrm{dd}, J=4.6,1.4,1 \mathrm{H}) ;{ }^{13} \mathrm{C} \mathrm{NMR}\left(75 \mathrm{MHz}, \mathrm{CDCl}_{3}\right) \delta 26.0,33.2,37.6,43.5,77.1$, $123.7,126.4,127.9,129.1,129.8,135.7,136.0,148.8,149.7,159.1,186.3$; MS m/z 320 $(\mathrm{M}+\mathrm{H})$; HRMS calcd. for $\left(\mathrm{C}_{20} \mathrm{H}_{21} \mathrm{~N}_{3} \mathrm{O}+\mathrm{H}\right) 320.1757$, found 320.1758 .

2-Butyl-3-(2-methoxy-ethyl)-1,3-diaza-spiro[4.4]non-1-en-4-one (3j). Product was purified by preparative HPLC (MACCEL 120-10-C18 column (50x20 mm) with a flow rate at $88 \mathrm{~mL} / \mathrm{min}$ utilizing an acetonitrile/water mobile phase and $0.1 \%$ trifluoroacetic acid as a modifier) as light yellow oil TFA salt (39 mg, 54\%); ${ }^{1} \mathrm{H}$ NMR (400 MHz, $\left.\mathrm{CDCl}_{3}\right) \delta 0.96(\mathrm{t}, J=7.2 \mathrm{~Hz}, 3 \mathrm{H}), 1.40-1.50(\mathrm{~m}, 2 \mathrm{H}), 1.73-1.81(\mathrm{~m}, 2 \mathrm{H}), 1.93-2.20$ $(\mathrm{m}, 8 \mathrm{H}), 2.95(\mathrm{t}, J=7.8 \mathrm{~Hz}, 2 \mathrm{H}), 3.33(\mathrm{~s}, 3 \mathrm{H}), 3.54(\mathrm{t}, J=5.0 \mathrm{~Hz}, 2 \mathrm{H}), 3.84(\mathrm{t}, J=5.0$ $\mathrm{Hz}, 2 \mathrm{H}) ;{ }^{13} \mathrm{C} \mathrm{NMR}\left(100 \mathrm{MHz}, \mathrm{CDCl}_{3}\right) \delta 13.3,22.2,25.8,27.1,27.6,37.3,41.5,59.2$, 68.9, 72.4, 172.2, 179.6; MS m/z $253(\mathrm{M}+\mathrm{H})$; HRMS calcd. for $\left(\mathrm{C}_{14} \mathrm{H}_{24} \mathrm{~N}_{2} \mathrm{O}_{2}+\mathrm{H}\right)$ 253.1910, found 253.1910 .

2-Butyl-3-isobutyl-1,3-diaza-spiro[4.4]non-1-en-4-one (3k). Product was purified by preparative HPLC (MACCEL 120-10-C18 column $(50 \times 20 \mathrm{~mm})$ with a flow rate at 88 $\mathrm{mL} / \mathrm{min}$ utilizing an acetonitrile/water mobile phase and $0.1 \%$ trifluoroacetic acid as a modifier) as light yellow oil TFA salt (36 mg, 50\%); ${ }^{1} \mathrm{H}$ NMR (400 MHz, $\left.\mathrm{CDCl}_{3}\right) \delta 0.95$ $(\mathrm{d}, J=6.4 \mathrm{~Hz}, 6 \mathrm{H}), 0.97(\mathrm{t}, J=7.0 \mathrm{~Hz}, 3 \mathrm{H}), 1.42-1.51(\mathrm{~m}, 2 \mathrm{H}), 1.75-1.83(\mathrm{~m}, 2 \mathrm{H})$, $1.93-2.20(\mathrm{~m}, 9 \mathrm{H}), 2.86(\mathrm{t}, J=8.0 \mathrm{~Hz}, 2 \mathrm{H}), 3.46(\mathrm{~d}, J=7.6 \mathrm{~Hz}, 2 \mathrm{H}) ;{ }^{13} \mathrm{C}$ NMR $(75$ $\left.\mathrm{MHz}, \mathrm{CDCl}_{3}\right) \delta 13.3,19.7,22.3,25.8,27.1,28.1,28.2,37.5,48.0,72.5,172.2,180.1$; MS $m / z 251(\mathrm{M}+\mathrm{H})$; HRMS calcd. for $\left(\mathrm{C}_{15} \mathrm{H}_{26} \mathrm{~N}_{2} \mathrm{O}+\mathrm{H}\right)$ 251.2118, found 251.2118. 


\section{SUPPORTING INFORMATION}

2-Butyl-3-m-tolyl-1,3-diaza-spiro[4.4]non-1-en-4-one (31). Product was purified by preparative HPLC (MACCEL 120-10-C18 column $(50 \times 20 \mathrm{~mm})$ with a flow rate at 88 $\mathrm{mL} / \mathrm{min}$ utilizing an acetonitrile/water mobile phase and $0.1 \%$ trifluoroacetic acid as a modifier) as light yellow oil TFA salt (55 mg, 69\%); ${ }^{1} \mathrm{H} \mathrm{NMR}\left(400 \mathrm{MHz}, \mathrm{CDCl}_{3}\right) \delta 0.82$ $(\mathrm{t}, J=7.4 \mathrm{~Hz}, 3 \mathrm{H}), 1.22-1.32(\mathrm{~m}, 2 \mathrm{H}), 1.47-1.55(\mathrm{~m}, 2 \mathrm{H}), 1.83-2.09(\mathrm{~m}, 8 \mathrm{H}), 2.32(\mathrm{t}$, $J=8.0 \mathrm{~Hz}, 2 \mathrm{H}), 2.40(\mathrm{~s}, 3 \mathrm{H}), 6.97-7.00(\mathrm{~m}, 2 \mathrm{H}), 7.21(\mathrm{~d}, J=7.6 \mathrm{~Hz}, 1 \mathrm{H}), 7.35(\mathrm{t}, J=$ 7.6 Hz, 1H); ${ }^{13} \mathrm{C}$ NMR (75 MHz, $\left.\mathrm{CDCl}_{3}\right) \delta 13.6,21.3,22.1,26.0,27.7,29.0,37.6,76.7$, 124.5, 128.1, 129.3, 129.4, 133.8, 139.6, 161.2, 185.8; MS m/z 285 (M+H); HRMS calcd. for $\left(\mathrm{C}_{18} \mathrm{H}_{24} \mathrm{~N}_{2} \mathrm{O}+\mathrm{H}\right) 285.1963$, found 285.1961.

2-Butyl-3-(4-methoxy-benzyl)-1,3-diaza-spiro[4.4]non-1-en-4-one (3m). Product was purified by preparative HPLC (MACCEL 120-10-C18 column (50x20 mm) with a flow rate at $88 \mathrm{~mL} / \mathrm{min}$ utilizing an acetonitrile/water mobile phase and $0.1 \%$ trifluoroacetic acid as a modifier) as light yellow oil TFA salt (41 mg, 48\%); ${ }^{1} \mathrm{H}$ NMR (400 MHz, $\left.\mathrm{CDCl}_{3}\right) \delta 0.87(\mathrm{t}, J=7.4 \mathrm{~Hz}, 3 \mathrm{H}), 1.28-1.37(\mathrm{~m}, 2 \mathrm{H}), 1.52-1.59(\mathrm{~m}, 2 \mathrm{H}), 1.77-2.01$ $(\mathrm{m}, 8 \mathrm{H}), 2.30(\mathrm{t}, J=8.0 \mathrm{~Hz}, 2 \mathrm{H}), 3.79(\mathrm{~s}, 3 \mathrm{H}), 4.61(\mathrm{~s}, 2 \mathrm{H}), 6.85(\mathrm{~d}, J=8.8 \mathrm{~Hz}, 2 \mathrm{H}), 7.09$ $(\mathrm{d}, J=8.8 \mathrm{~Hz}, 2 \mathrm{H}) ;{ }^{13} \mathrm{C} \mathrm{NMR}\left(100 \mathrm{MHz}, \mathrm{CDCl}_{3}\right) \delta 13.7,22.3,26.1,27.7,28.9,37.3$, 43.0, 55.3, 76.5, 114.2, 128.1, 128.7, 159.1, 161.8, 186.8; MS m/z $315(\mathrm{M}+\mathrm{H})$; HRMS calcd. for $\left(\mathrm{C}_{19} \mathrm{H}_{26} \mathrm{~N}_{2} \mathrm{O}_{2}+\mathrm{H}\right) 315.2065$, found 315.2067.

\section{4'-(2-Butyl-4-oxo-1,3-diaza-spiro[4.4]non-1-en-3-ylmethyl)-biphenyl-2-carbonitrile}

(3n). Product was purified by prep-TLC (ethyl acetate/hexane $=1 / 1$ ) as a yellow solid $(58 \mathrm{mg}, 75 \%) ;{ }^{1} \mathrm{H}$ NMR (400 MHz, $\left.\mathrm{CDCl}_{3}\right) \delta 0.87(\mathrm{t}, J=7.2 \mathrm{~Hz}, 3 \mathrm{H}), 1.30-1.39(\mathrm{~m}$, 2H), $1.57-1.64(\mathrm{~m}, 2 \mathrm{H}), 1.80-2.07(\mathrm{~m}, 8 \mathrm{H}), 2.40(\mathrm{t}, J=7.8 \mathrm{~Hz}, 2 \mathrm{H}), 4.77(\mathrm{~s}, 2 \mathrm{H}), 7.28$ $(\mathrm{d}, J=8.0 \mathrm{~Hz}, 2 \mathrm{H}), 7.44-7.50(\mathrm{~m}, 2 \mathrm{H}), 7.53-7.57(\mathrm{~m}, 2 \mathrm{H}), 7.65(\mathrm{dt}, J=7.6,1.5 \mathrm{~Hz}$, 


\section{SUPPORTING INFORMATION}

1H), $7.77(\mathrm{dd}, J=7.6,1.6 \mathrm{~Hz}, 1 \mathrm{H}) ;{ }^{13} \mathrm{C} \mathrm{NMR}\left(75 \mathrm{MHz}, \mathrm{CDCl}_{3}\right) \delta 13.6,22.2,26.0,27.7$, $28.6,37.4,43.3,76.2,111.2,118.5,127.0,127.7,129.4,129.9,132.8,133.7,136.8$, 137.8, 144.6, 162.5, 186.0; MS m/z $386(\mathrm{M}+\mathrm{H})$; HRMS calcd. for $\left(\mathrm{C}_{25} \mathrm{H}_{27} \mathrm{~N}_{3} \mathrm{O}+\mathrm{H}\right)$ 386.2219, found 386.2227.

2-Butyl-3-pyridin-2-ylmethyl-1,3-diaza-spiro[4.4]non-1-en-4-one (3o). Product was purified by preparative HPLC (MACCEL 120-10-C18 column (50x20 mm) with a flow rate at $88 \mathrm{~mL} / \mathrm{min}$ utilizing an acetonitrile/water mobile phase and $0.1 \%$ trifluoroacetic acid as a modifier) as light yellow oil TFA salt (34 mg, 43\%); ${ }^{1} \mathrm{H}$ NMR (400 MHz, $\left.\mathrm{CDCl}_{3}\right) \delta 0.86(\mathrm{t}, J=7.4 \mathrm{~Hz}, 3 \mathrm{H}), 1.29-1.38(\mathrm{~m}, 2 \mathrm{H}), 1.54-1.62(\mathrm{~m}, 2 \mathrm{H}), 1.82-2.04$ $(\mathrm{m}, 8 \mathrm{H}), 2.42(\mathrm{t}, J=7.8 \mathrm{~Hz}, 2 \mathrm{H}), 4.81(\mathrm{~s}, 2 \mathrm{H}), 7.13(\mathrm{~d}, J=7.8 \mathrm{~Hz}, 1 \mathrm{H}), 7.19-7.23(\mathrm{~m}$, 1H), $7.66(\mathrm{dt}, J=7.8,1.7 \mathrm{~Hz}, 1 \mathrm{H}), 8.53-8.55(\mathrm{~m}, 1 \mathrm{H}) ;{ }^{13} \mathrm{C} \mathrm{NMR}\left(75 \mathrm{MHz}, \mathrm{CDCl}_{3}\right) \delta$ $13.6,22.2,26.0,27.7,28.6,37.3,45.5,76.3,121.2,122.7,137.0,149.5,155.9,162.3$, 186.3; MS m/z $286(\mathrm{M}+\mathrm{H})$; HRMS calcd. for $\left(\mathrm{C}_{17} \mathrm{H}_{23} \mathrm{~N}_{3} \mathrm{O}+\mathrm{H}\right) 286.1913$, found 286.1914. 2-Butyl-3-furan-2-ylmethyl-1,3-diaza-spiro[4.4]non-1-en-4-one (3p). Product was purified by preparative HPLC (MACCEL 120-10-C18 column $(50 \times 20 \mathrm{~mm})$ with a flow rate at $88 \mathrm{~mL} / \mathrm{min}$ utilizing an acetonitrile/water mobile phase and $0.1 \%$ trifluoroacetic acid as a modifier) as light yellow oil TFA salt (28 mg, 36\%); ${ }^{1} \mathrm{H}$ NMR (400 MHz, $\left.\mathrm{CDCl}_{3}\right) \delta 0.94(\mathrm{t}, J=7.4 \mathrm{~Hz}, 3 \mathrm{H}), 1.38-1.48(\mathrm{~m}, 2 \mathrm{H}), 1.66-1.73(\mathrm{~m}, 2 \mathrm{H}), 1.94-2.05$ $(\mathrm{m}, 8 \mathrm{H}), 2.84(\mathrm{t}, J=7.8 \mathrm{~Hz}, 2 \mathrm{H}), 4.78(\mathrm{~s}, 2 \mathrm{H}), 6.34-6.38(\mathrm{~m}, 2 \mathrm{H}), 7.38-7.39(\mathrm{~m}, 1 \mathrm{H})$; ${ }^{13} \mathrm{C}$ NMR $\left(75 \mathrm{MHz}, \mathrm{CDCl}_{3}\right) \delta 13.4,22.3,25.8,27.5,27.8,37.2,37.4,73.6,109.6,110.9$, 143.3, 147.1, 162.0, 168.8; MS m/z $275(\mathrm{M}+\mathrm{H})$; HRMS calcd. for $\left(\mathrm{C}_{16} \mathrm{H}_{22} \mathrm{~N}_{2} \mathrm{O}_{2}+\mathrm{H}\right)$ 275.1754 , found 275.1754 


\section{SUPPORTING INFORMATION}

3-(1-Benzyl-pyrrolidin-3-yl)-2-butyl-1,3-diaza-spiro[4.4]non-1-en-4-one

(3q).

Product was purified by preparative HPLC (MACCEL 120-10-C18 column (50x20 mm)

with a flow rate at $88 \mathrm{~mL} / \mathrm{min}$ utilizing an acetonitrile/water mobile phase and $0.1 \%$ trifluoroacetic acid as a modifier) as light yellow oil (73 mg, 78\%); ${ }^{1} \mathrm{H}$ NMR (400 MHz, $\left.\mathrm{CDCl}_{3}\right) \delta 0.94(\mathrm{t}, J=7.2 \mathrm{~Hz}, 3 \mathrm{H}), 1.36-1.45(\mathrm{~m}, 2 \mathrm{H}), 1.60-1.68(\mathrm{~m}, 4 \mathrm{H}), 1.69-1.96$ $(\mathrm{m}, 6 \mathrm{H}), 2.09-2.24(\mathrm{~m}, 2 \mathrm{H}), 2.49-2.54(\mathrm{~m}, 2 \mathrm{H}), 2.74-2.92(\mathrm{~m}, 4 \mathrm{H}), 3.66-3.73(\mathrm{~m}$, 2H), $4.39-4.47(\mathrm{~m}, 1 \mathrm{H}), 7.26-7.35(\mathrm{~m}, 5 \mathrm{H}) ;{ }^{13} \mathrm{C} \mathrm{NMR}\left(100 \mathrm{MHz}, \mathrm{CDCl}_{3}\right) \delta 13.8,22.4$, $26.1,28.4,28.8,29.1,37.3,37.4,50.9,53.6,56.0,60.1,76.1,127.3,128.4,128.9,161.9$, 186.9; MS m/z $354(\mathrm{M}+\mathrm{H})$; HRMS calcd. for $\left(\mathrm{C}_{22} \mathrm{H}_{31} \mathrm{~N}_{3} \mathrm{O}+\mathrm{H}\right)$ 354.2536, found 354.2540.

\section{2-Butyl-3-[2'-(1H-tetrazol-5-yl)-biphenyl-4-ylmethyl]-1,3-diaza-spiro[4.4]non-1-en-}

4-one (2). Product was purified by prep-TLC (ethyl acetate/hexane/methanol = 45/45/10) as light yellow solid (62 mg, 73\%); ${ }^{1} \mathrm{H}$ NMR (400 MHz, DMSO-d 6 ) $\delta 0.80(\mathrm{t}, J=7.4 \mathrm{~Hz}$, $3 \mathrm{H}$ ), $1.21-1.31$ (sextet, $J=7.3 \mathrm{~Hz}, 2 \mathrm{H}$ ), $1.43-1.51$ (quintet, $J=7.5 \mathrm{~Hz}, 2 \mathrm{H}$ ), $1.62-$ $1.88(\mathrm{~m}, 8 \mathrm{H}), 2.29(\mathrm{t}, J=7.6 \mathrm{~Hz}, 2 \mathrm{H}), 4.67(\mathrm{~s}, 2 \mathrm{H}), 7.03-7.09(\mathrm{~m}, 4 \mathrm{H}), 7.52-7.66(\mathrm{~m}$,

4H) (The ${ }^{1} \mathrm{H}$ NMR spectrum was in accord with that reported in literature $\left.4 \mathrm{~b}.\right) ;{ }^{13} \mathrm{C}$ NMR $\left(100 \mathrm{MHz}, \mathrm{DMSO}-\mathrm{d}_{6}\right) \delta 13.9,21.7,25.6,26.8,27.7,36.9,42.5,76.0,125.8,127.2$, $128.4,129.6,130.3,130.4,130.7,135.3,140.4,140.6,159.5,161.3,185.9 ;$ MS m/z 429 $(\mathrm{M}+\mathrm{H})$; HRMS calcd. for $\left(\mathrm{C}_{25} \mathrm{H}_{28} \mathrm{~N}_{6} \mathrm{O}+\mathrm{H}\right)$ 429.2397, found 429.2392. 


\section{SUPPORTING INFORMATION}

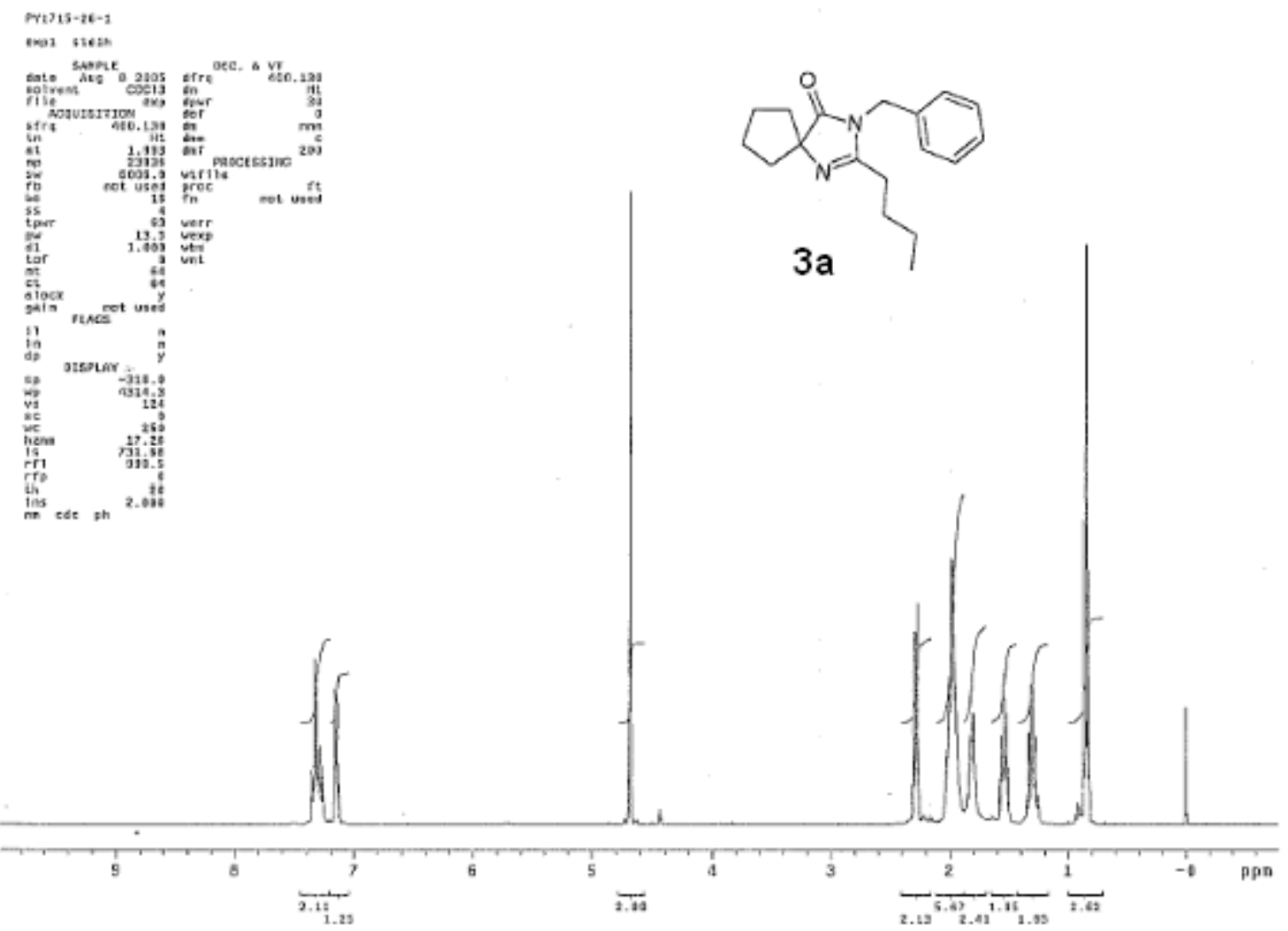


SUPPORTING INFORMATION

tye gasexv:

Puise setuencet sapui

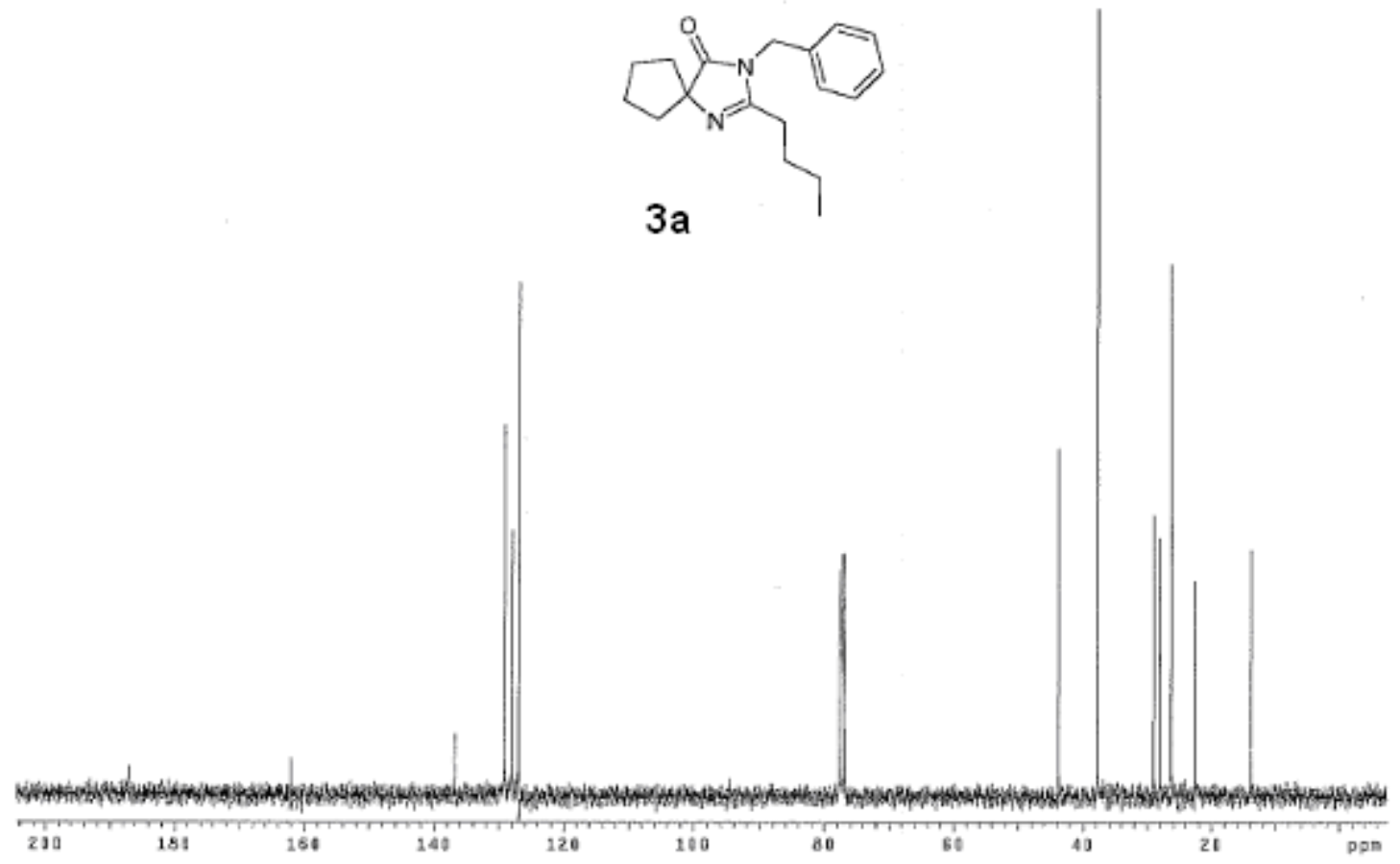




\section{SUPPORTING INFORMATION}
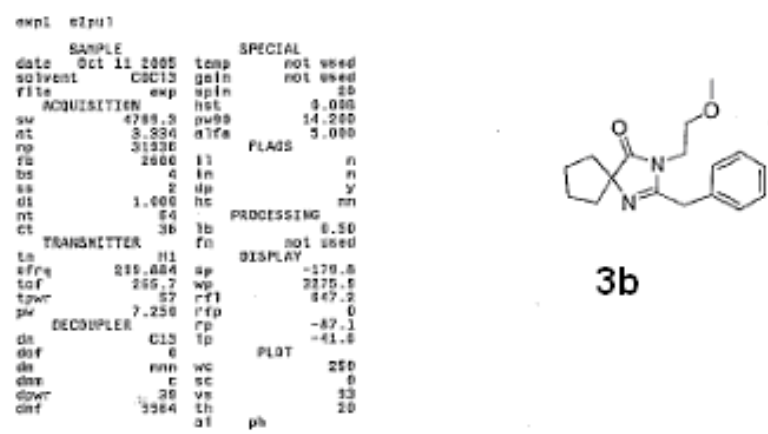

$3 b$

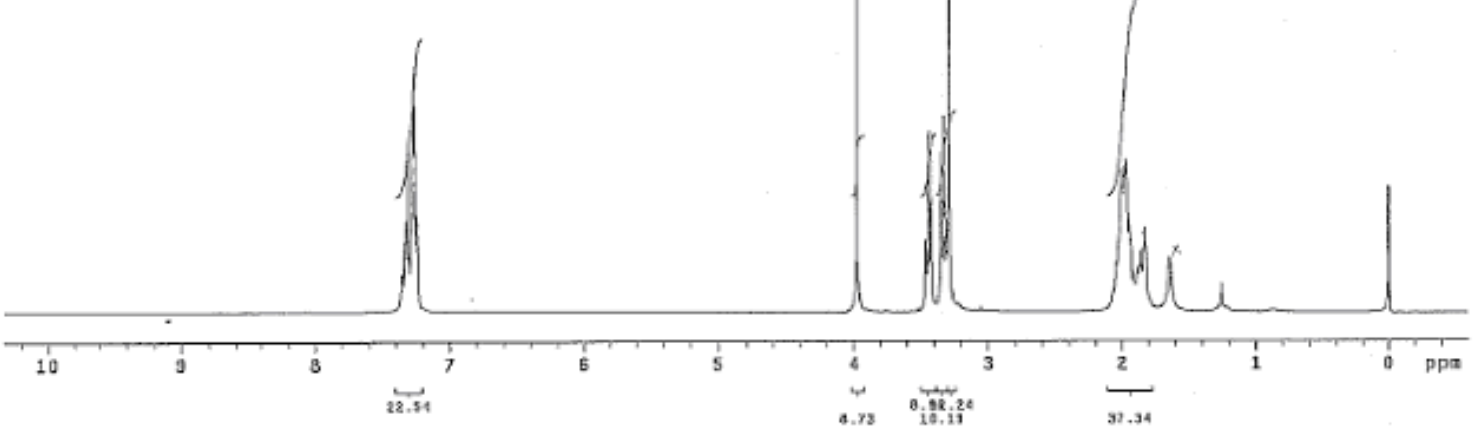




\section{SUPPORTING INFORMATION}
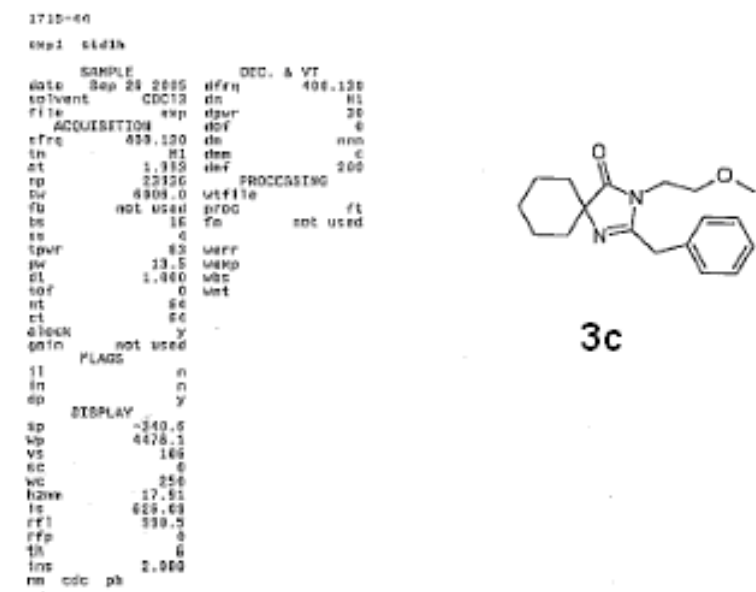

$3 c$

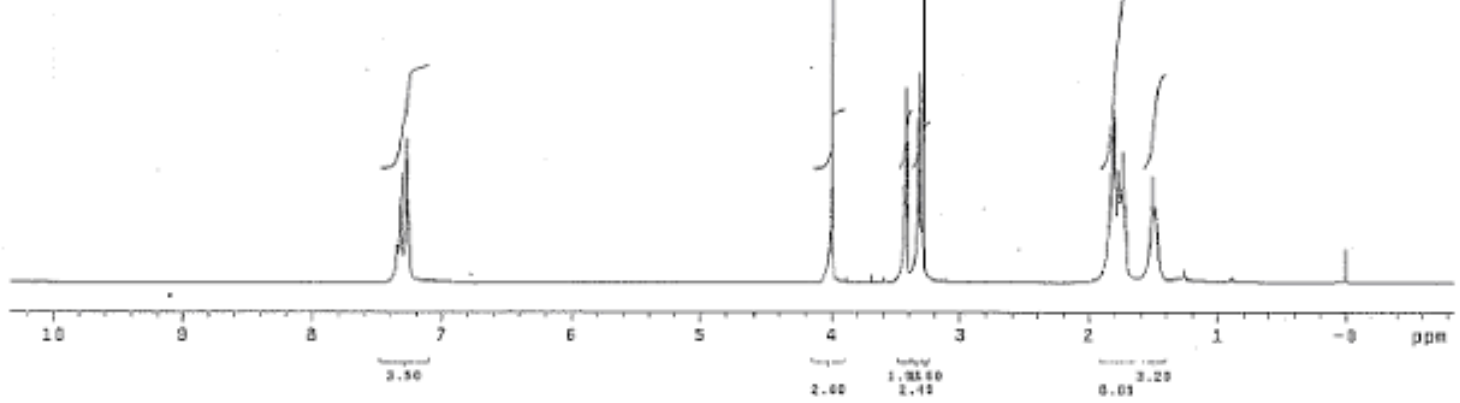


SUPPORTING INFORMATION

1719-44-C13

nulse seguence: sêpo1

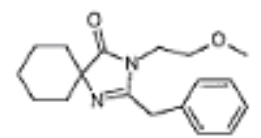

$3 c$

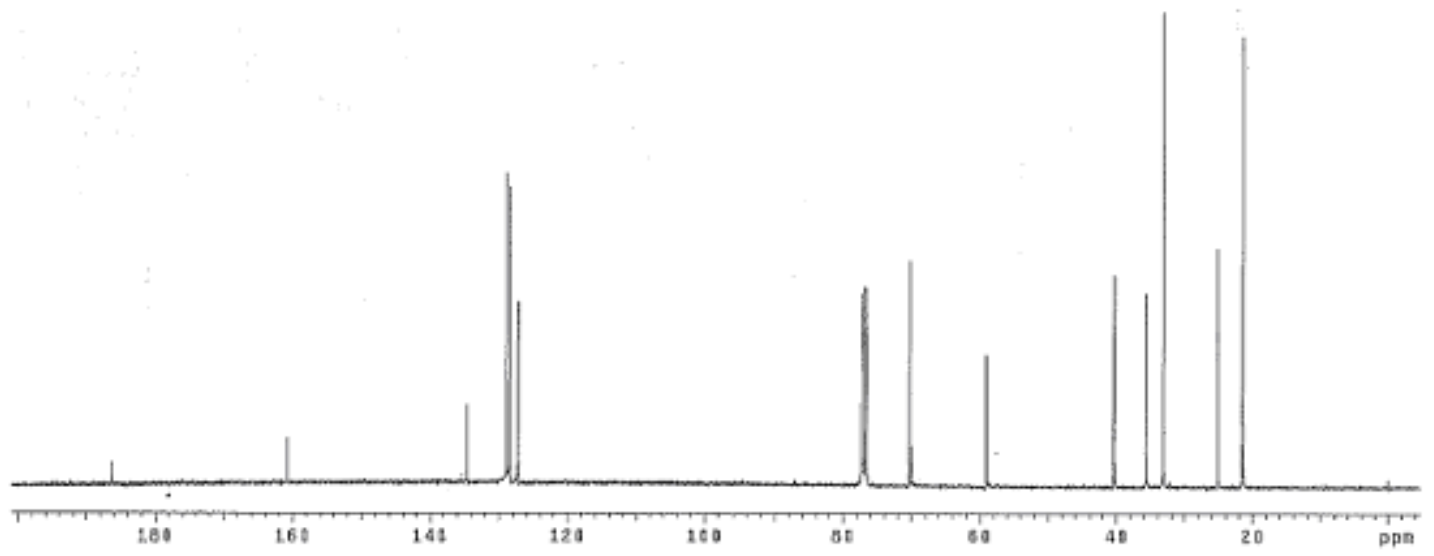




\section{SUPPORTING INFORMATION}

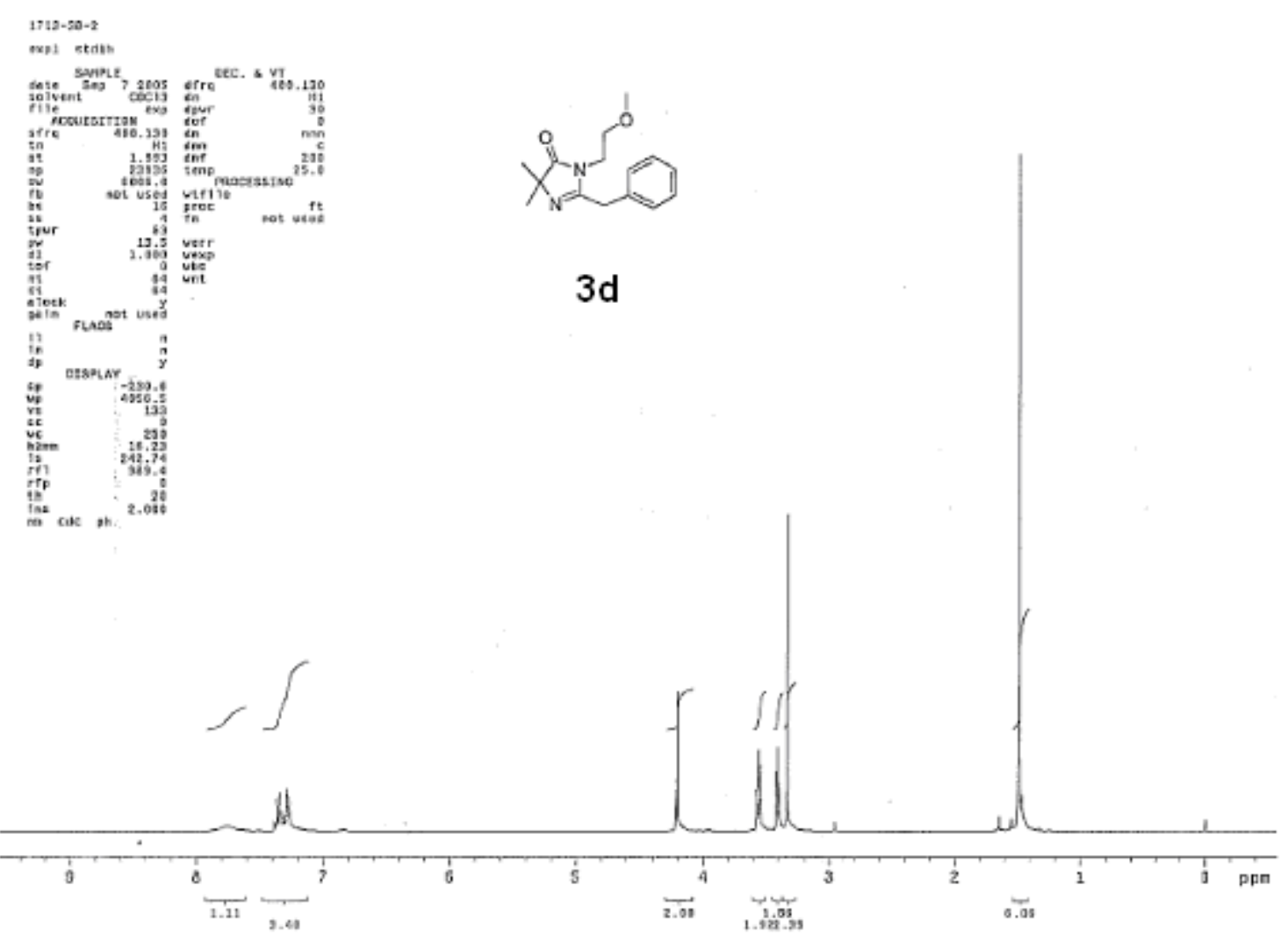




\section{SUPPORTING INFORMATION}
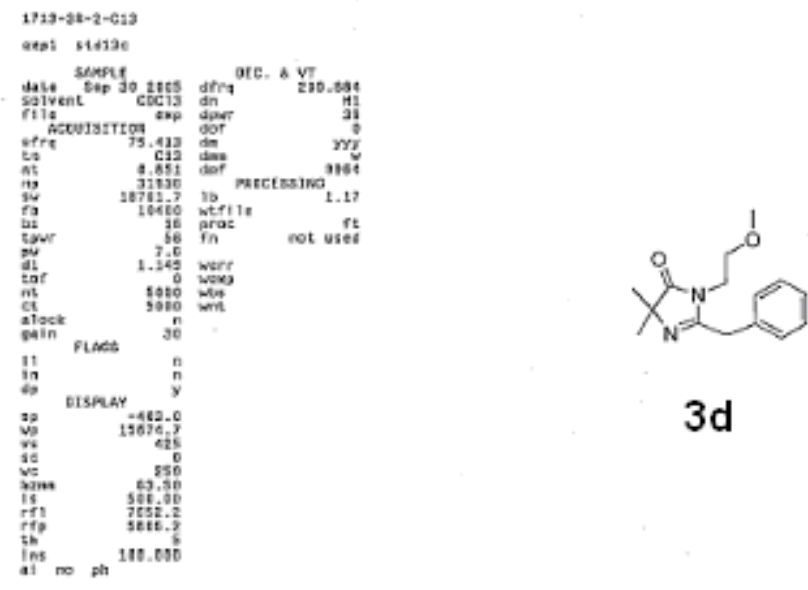

$3 d$

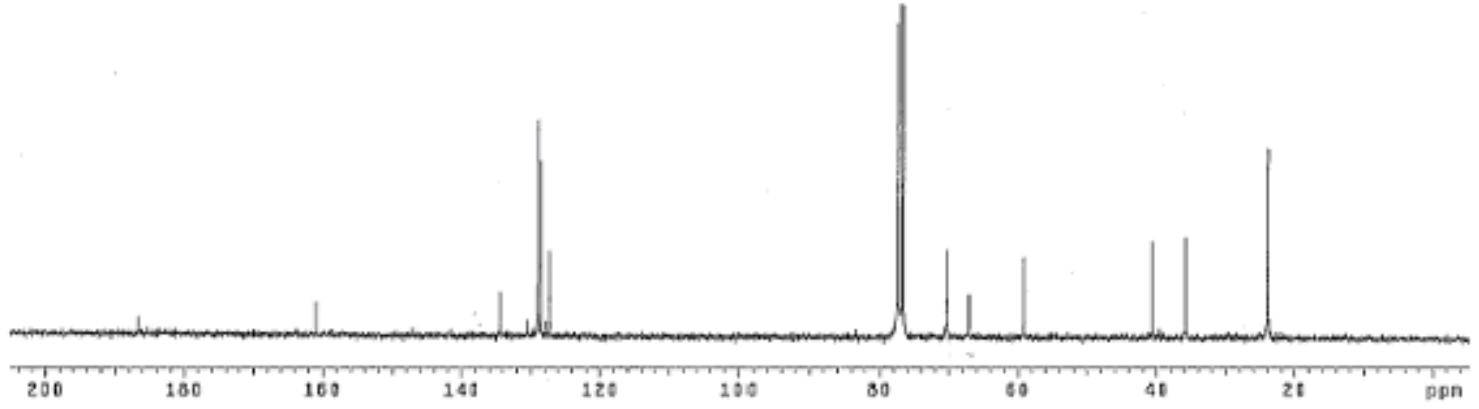




\section{SUPPORTING INFORMATION}
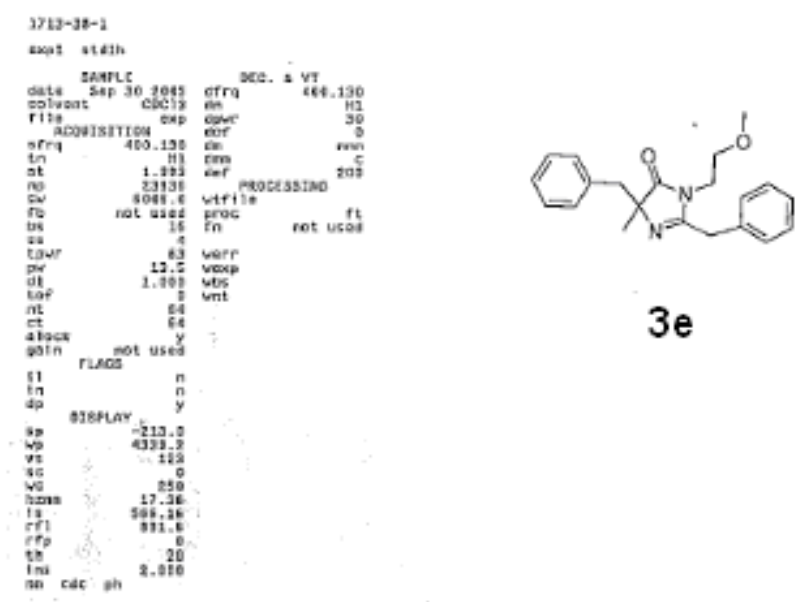

$3 e$

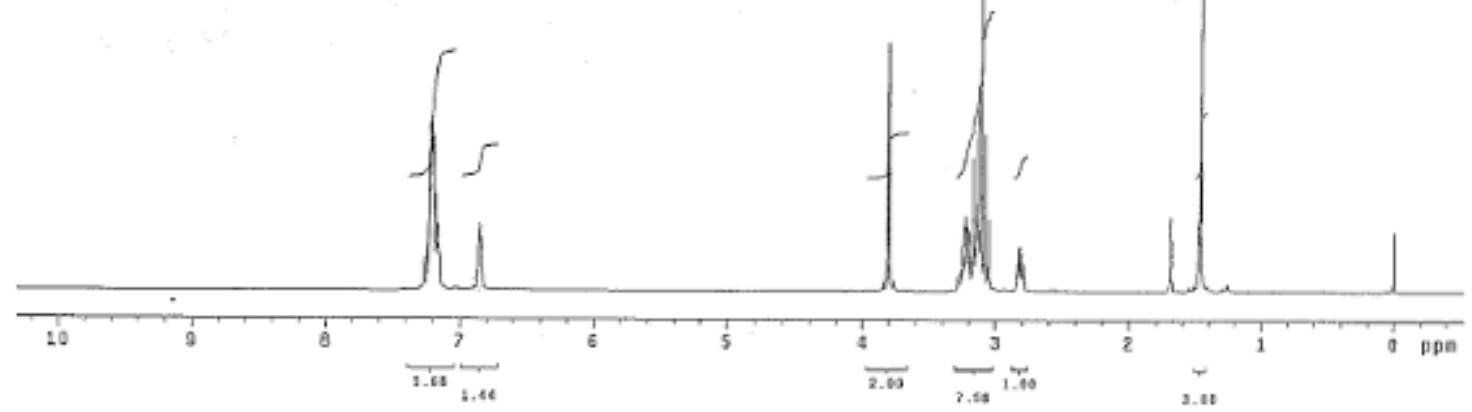


SUPPORTING INFORMATION

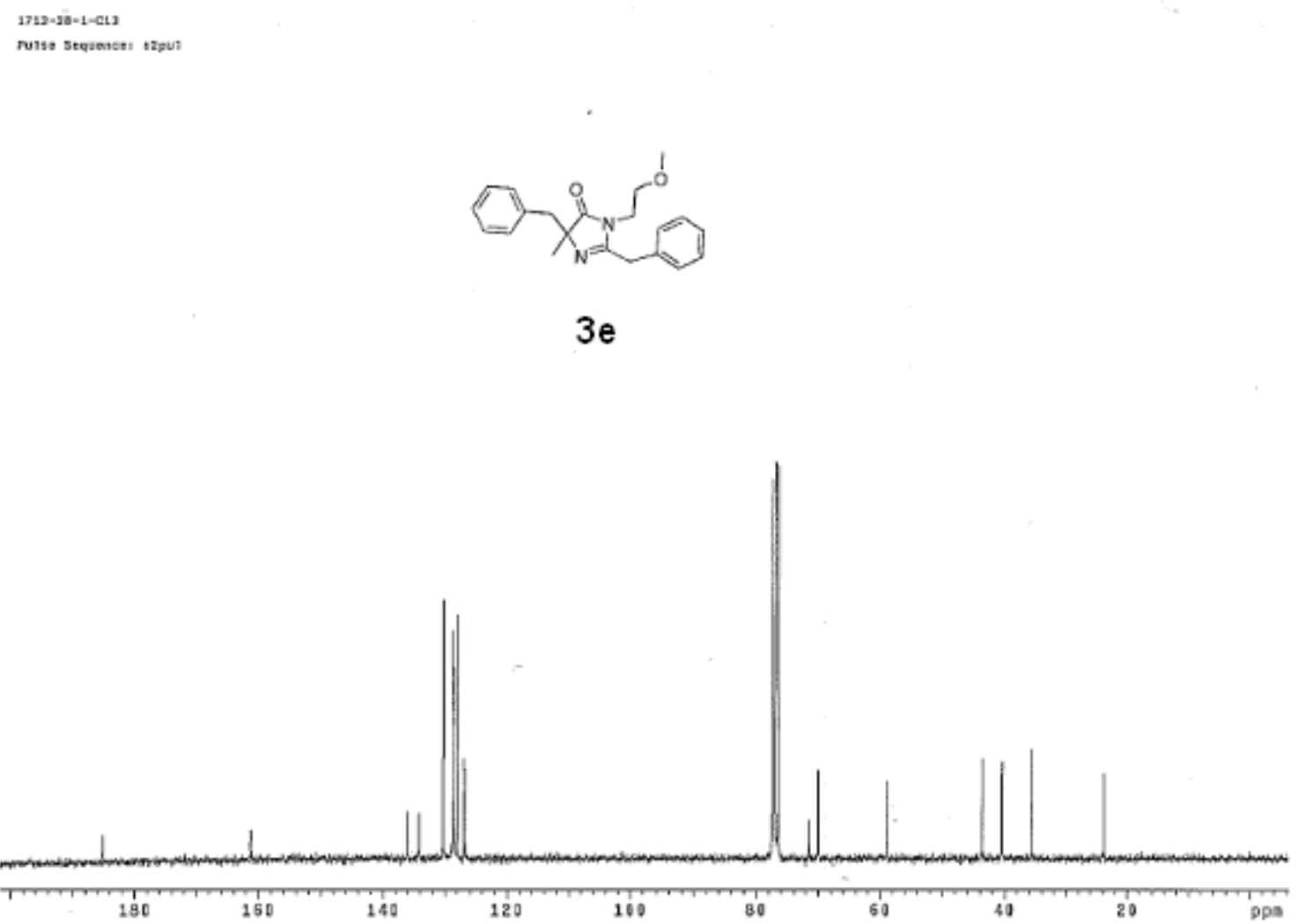




\section{SUPPORTING INFORMATION}
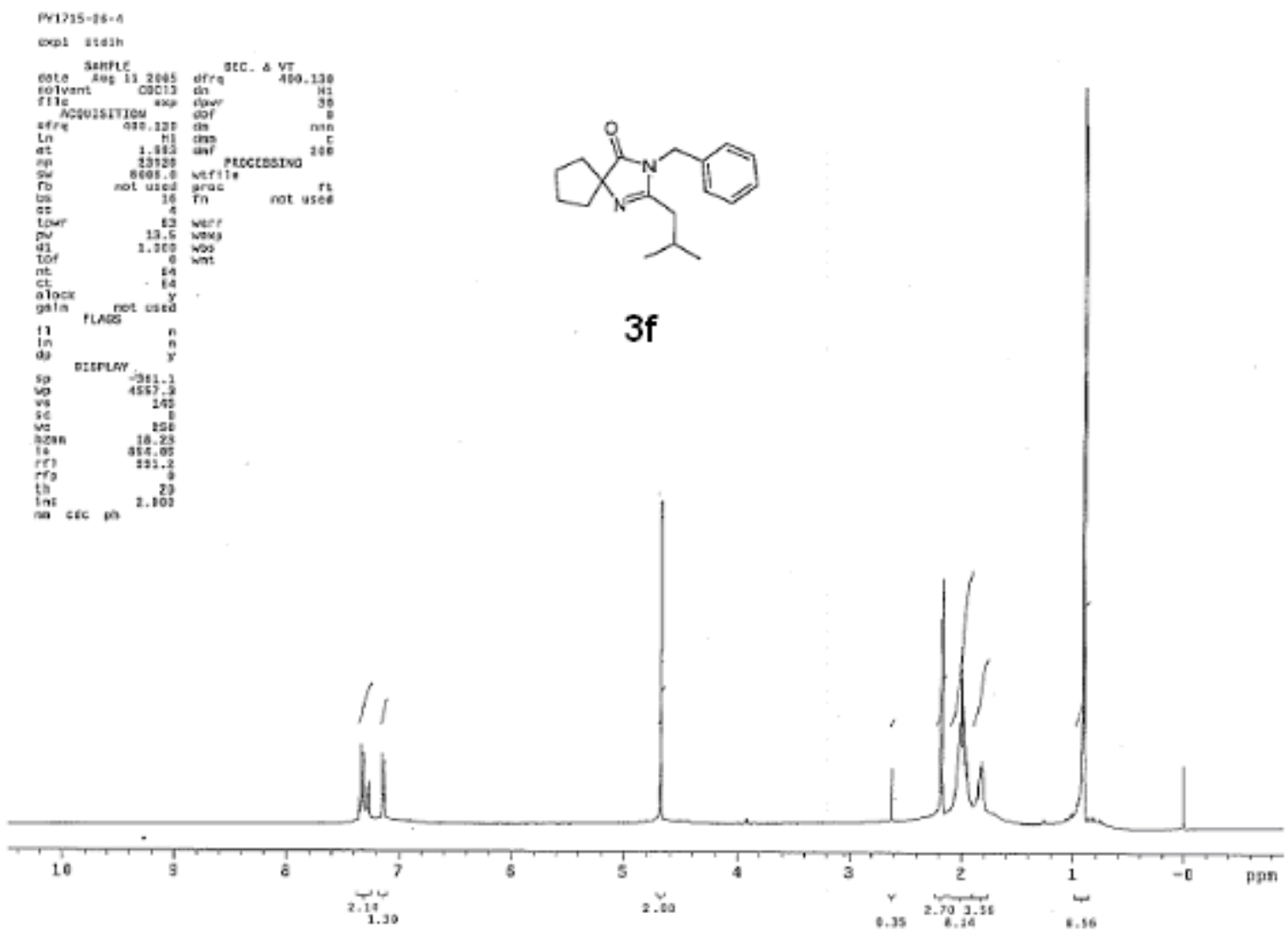
SUPPORTING INFORMATION

Fr1715-21-8-C12

Pulas sefiences stapt

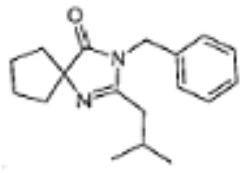

$3 f$

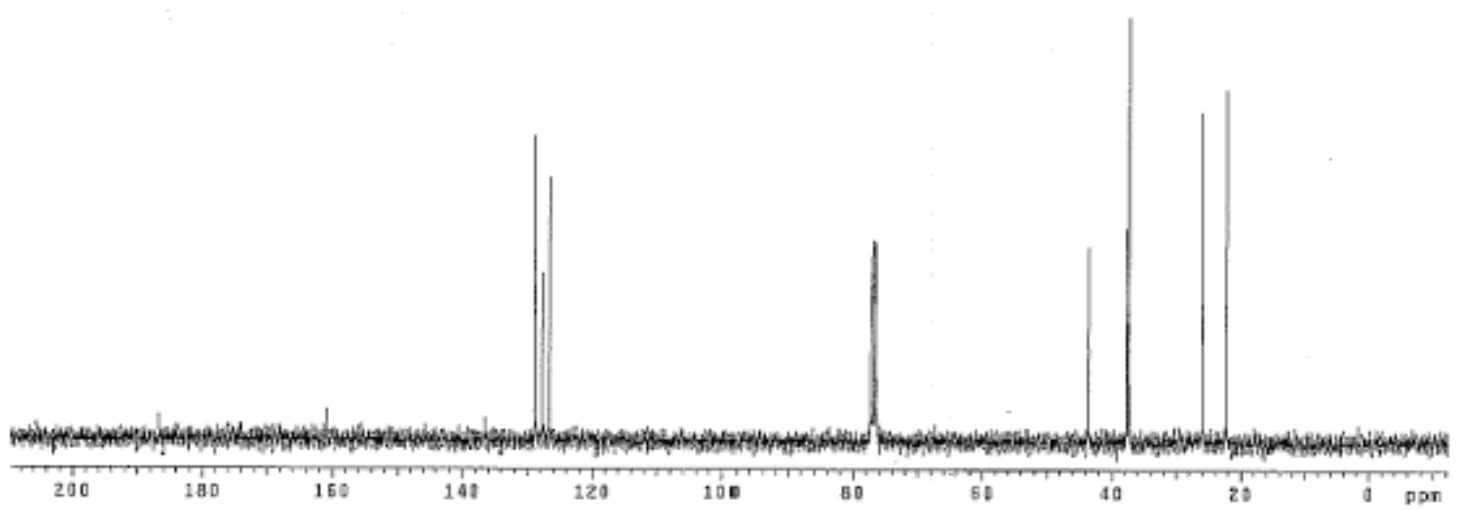




\section{SUPPORTING INFORMATION}
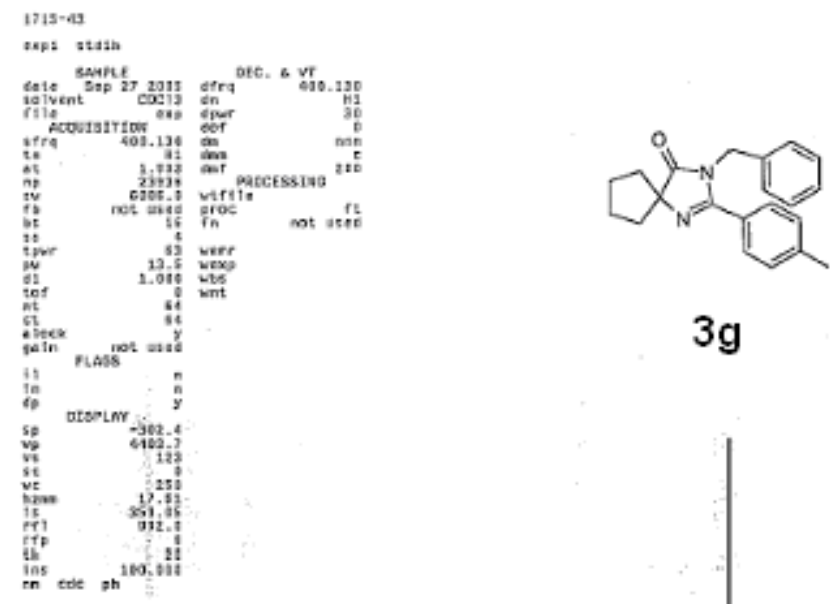

$3 g$

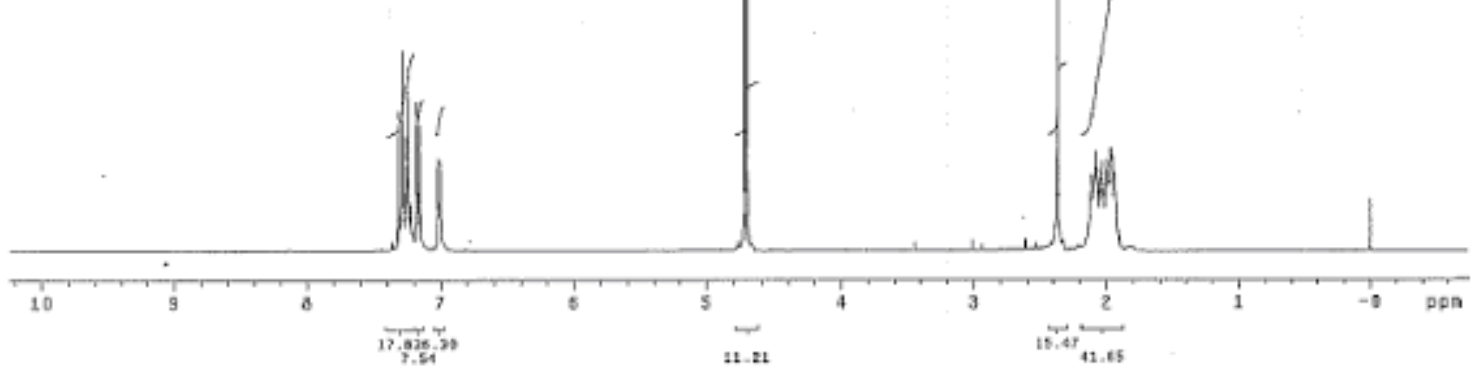


SUPPORTING INFORMATION

$1713-23-\mathrm{C} 18$

Calse ateconcet 120

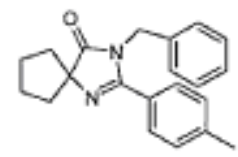

$3 \mathrm{~g}$

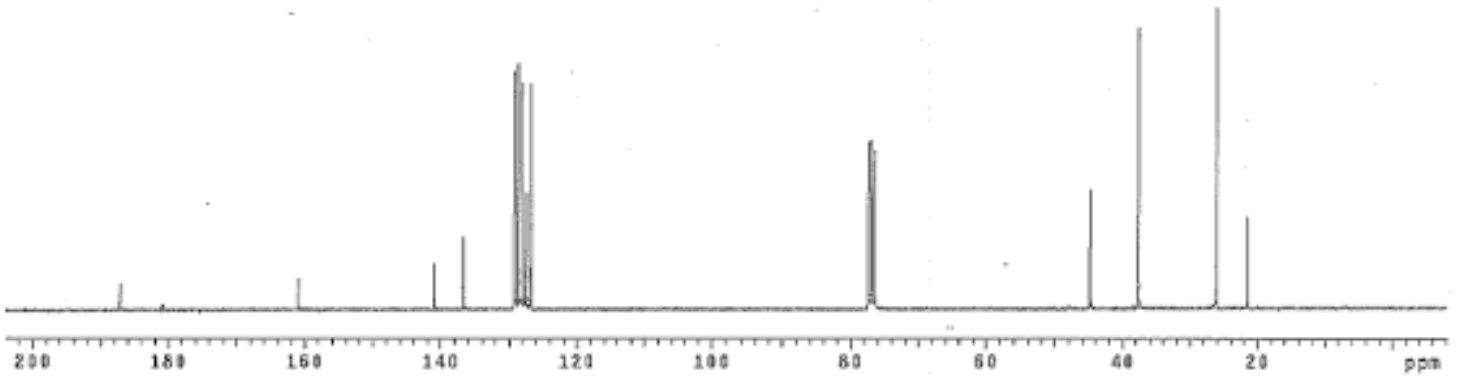




\section{SUPPORTING INFORMATION}

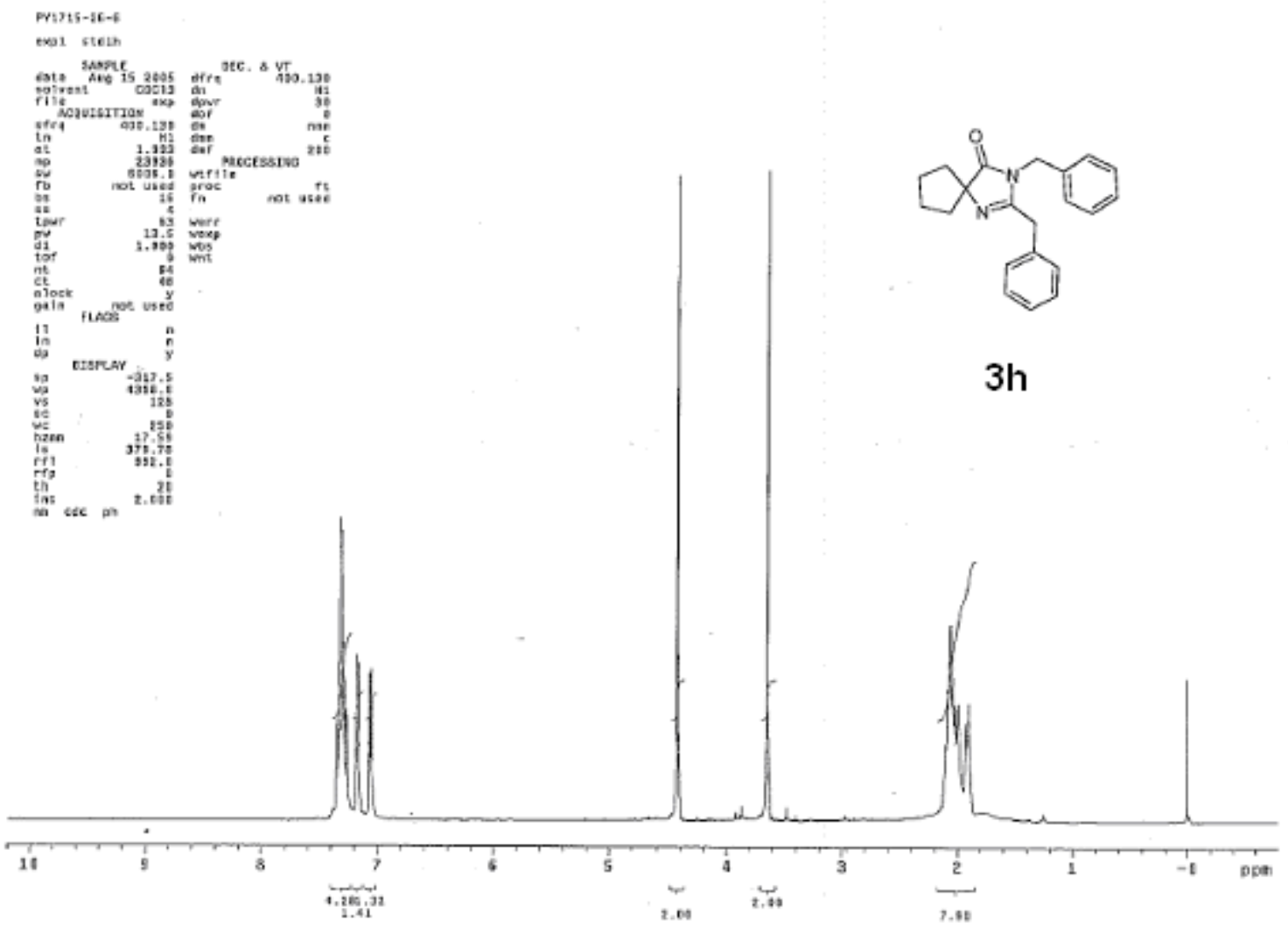


SUPPORTING INFORMATION

Prins-2t-4-6.13

puls setuencel s2se

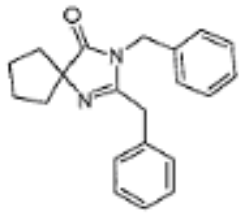

3h

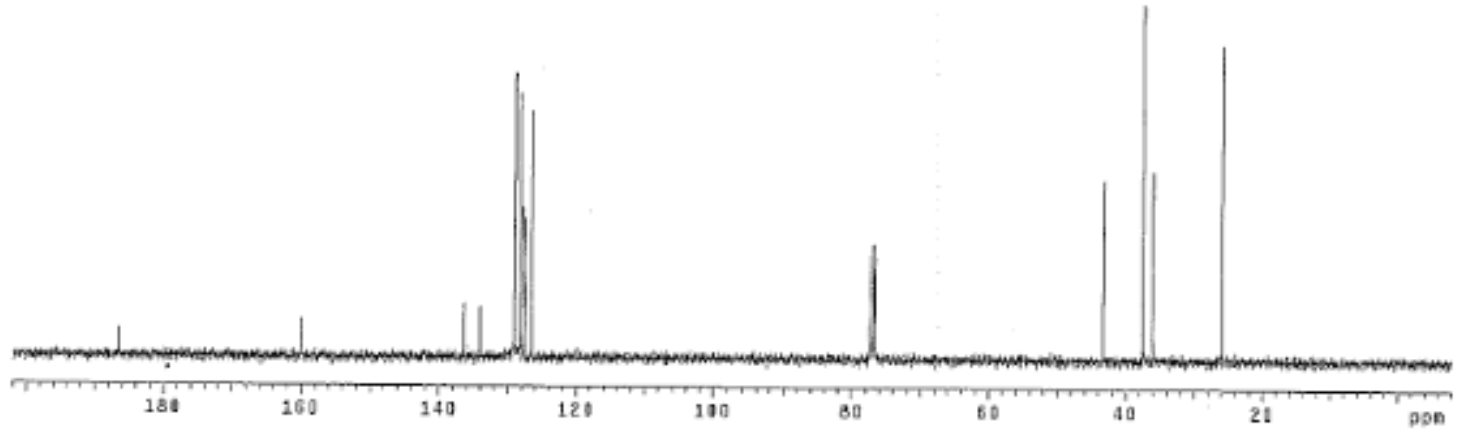




\section{SUPPORTING INFORMATION}
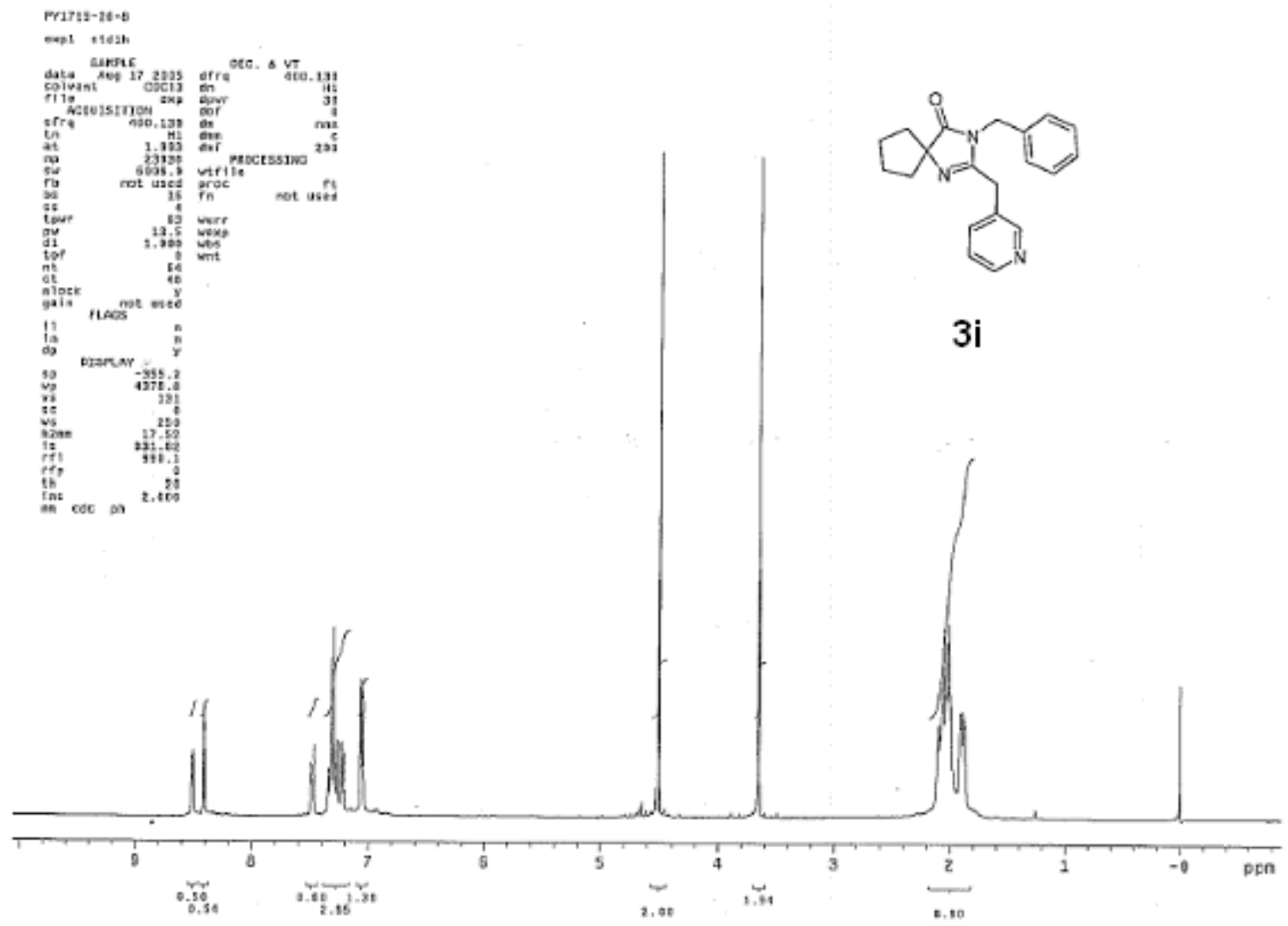
SUPPORTING INFORMATION

m1715-25-8-013

Pelse Benween stou

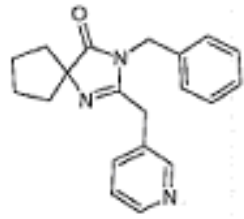

$3 \mathbf{i}$

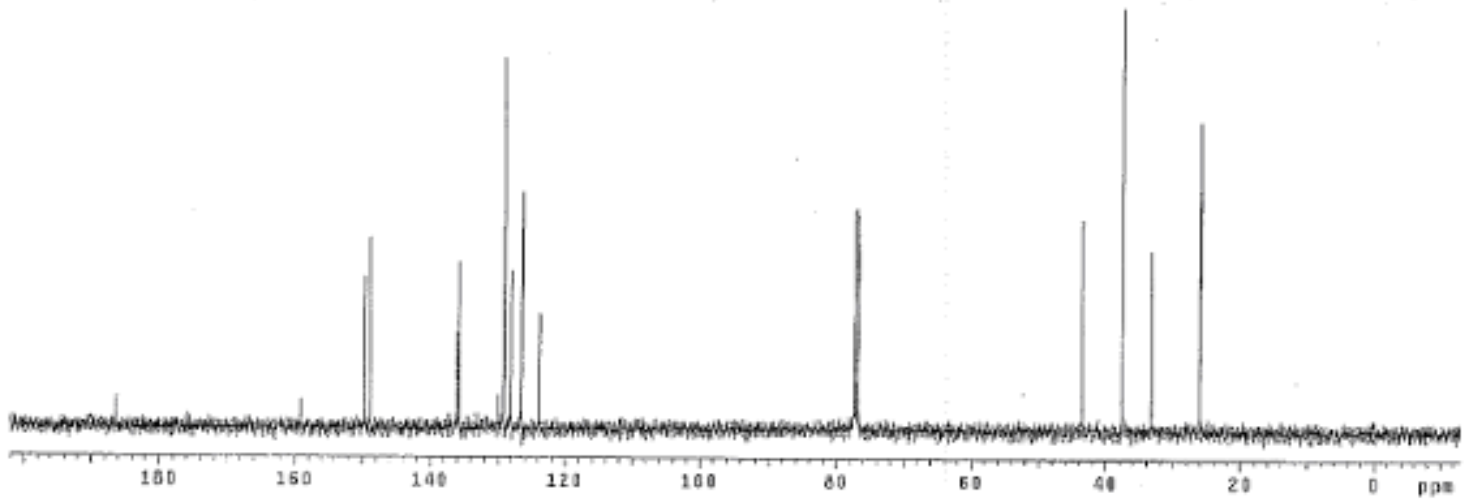




\section{SUPPORTING INFORMATION}

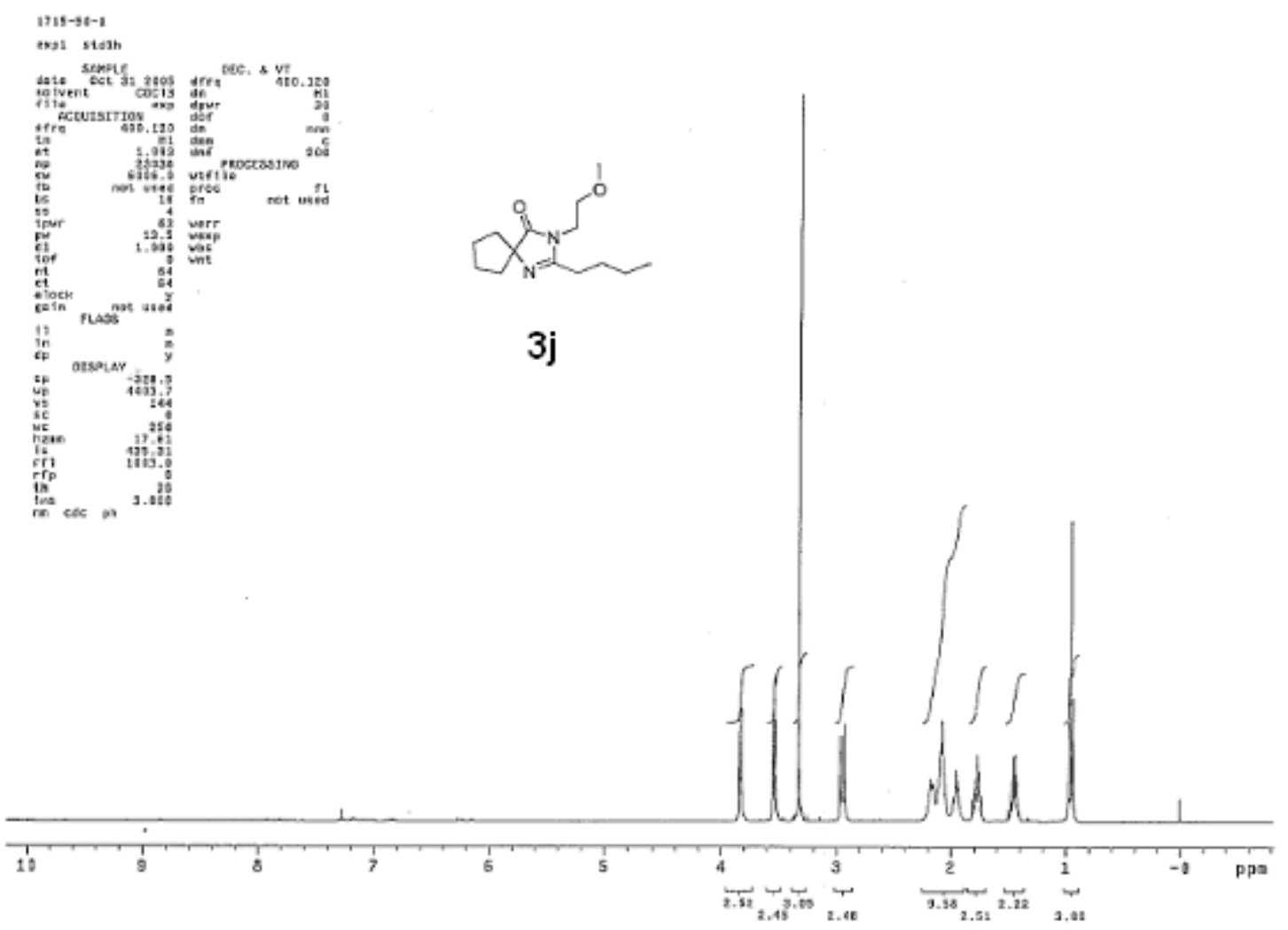


SUPPORTING INFORMATION

$5315-64-3-613$

Putse socuaneat c2gu)

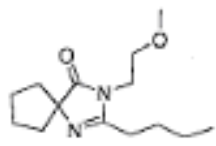

3j

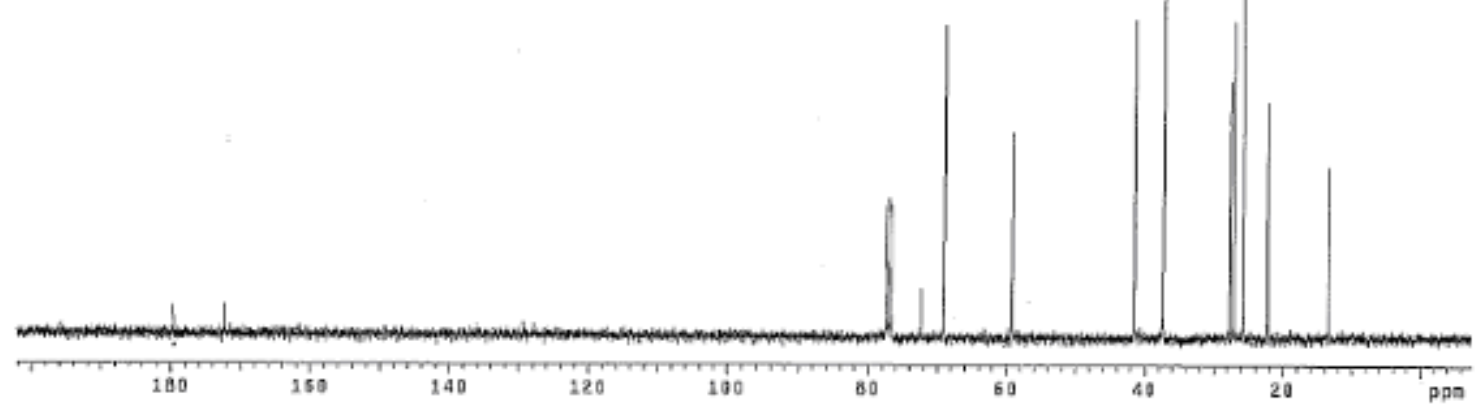




\section{SUPPORTING INFORMATION}
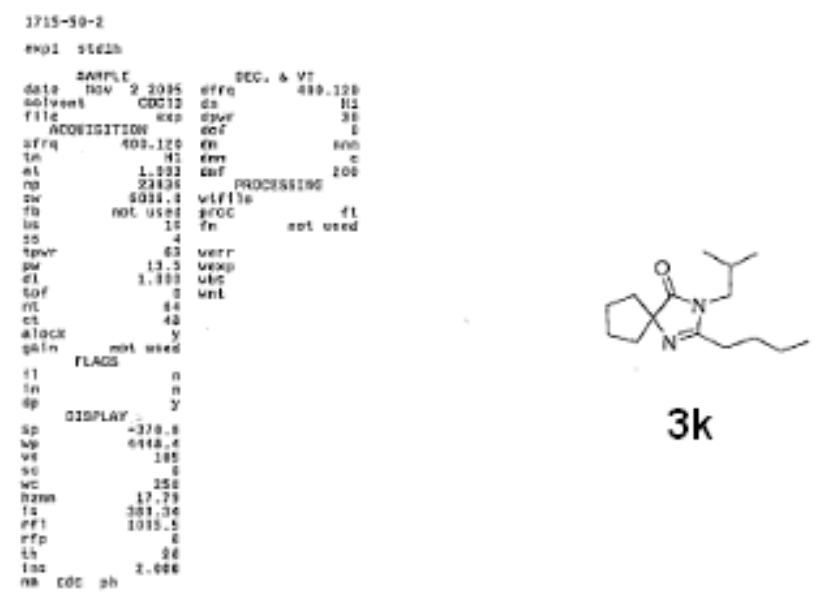

3k

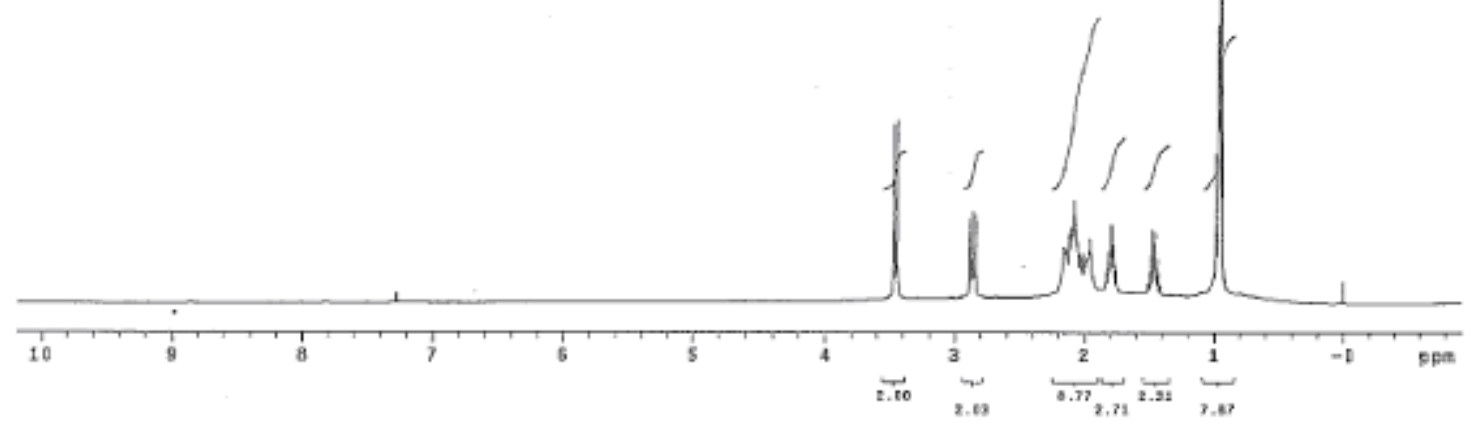


SUPPORTING INFORMATION

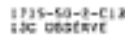

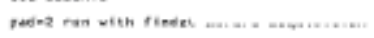

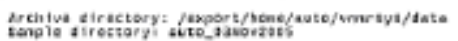

pese sugeente: stov1

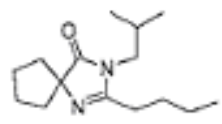

3k

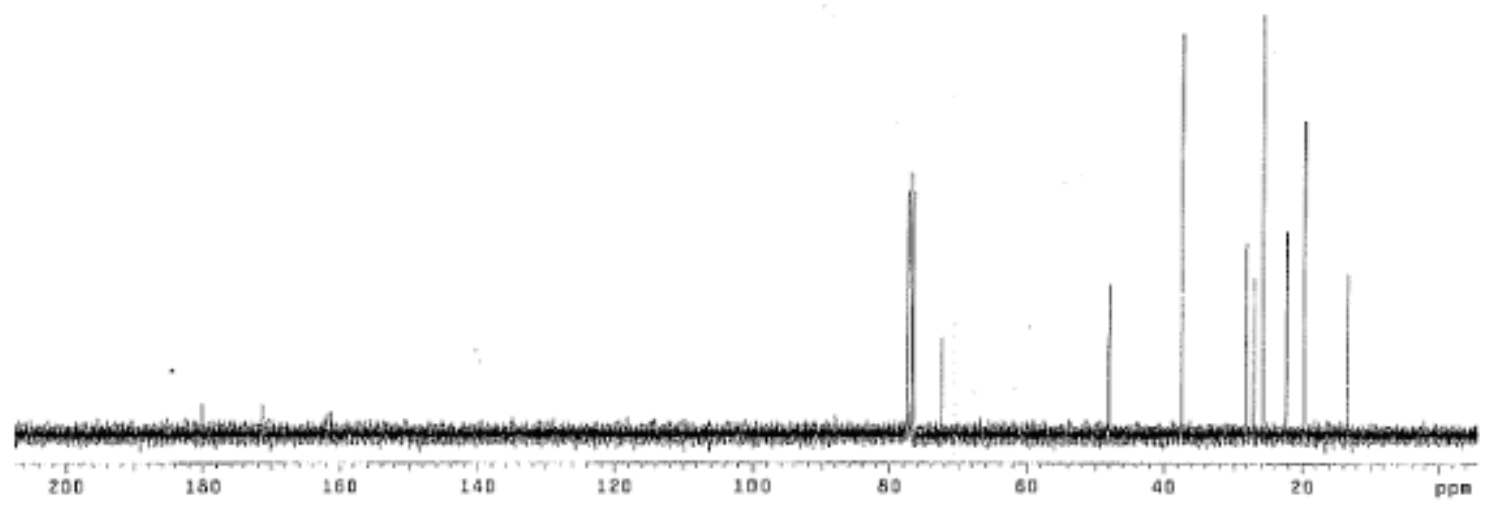




\section{SUPPORTING INFORMATION}

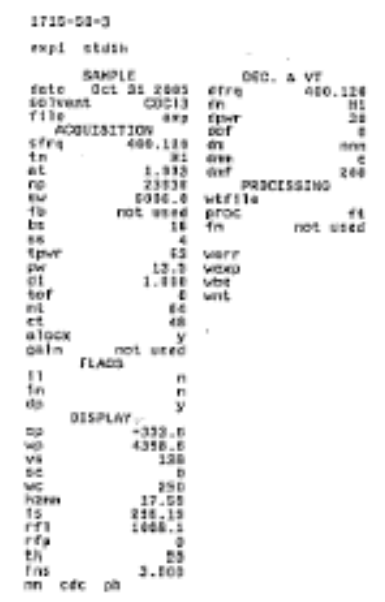

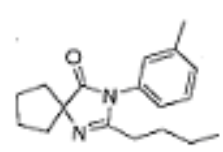

3।

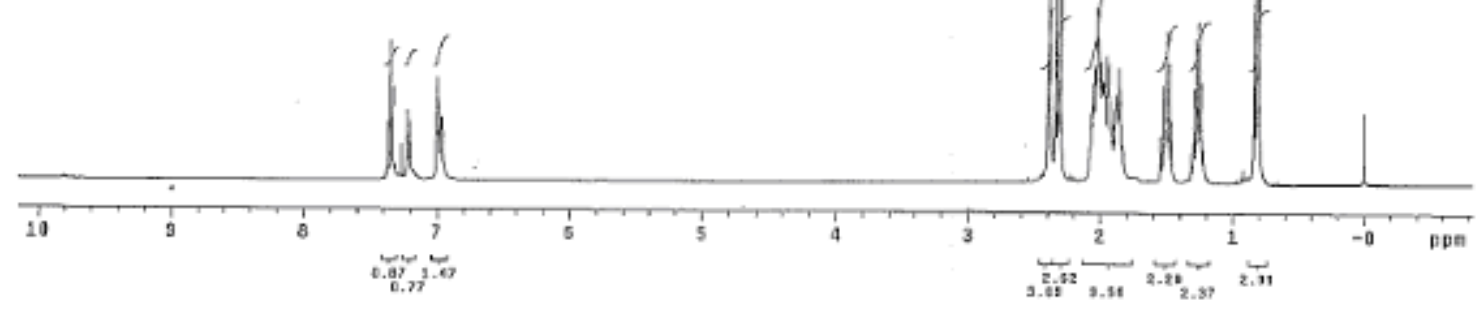




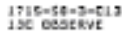

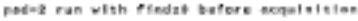

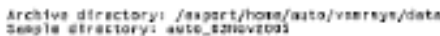

ralse Detuente: stpul

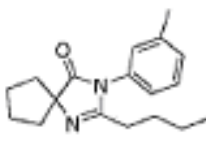

3।

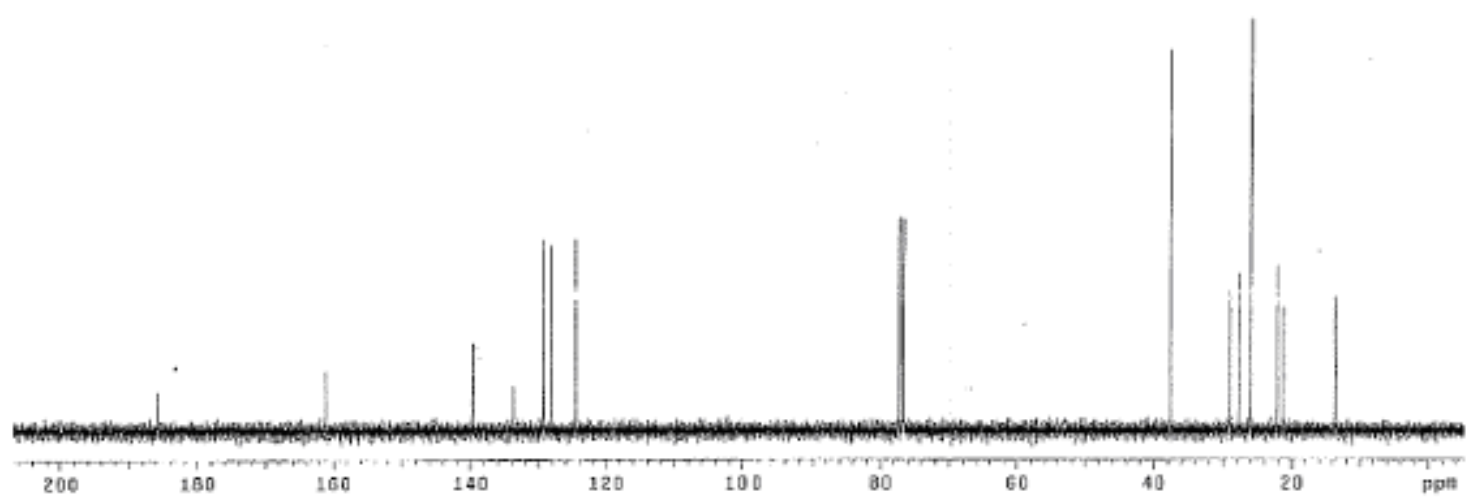




\section{SUPPORTING INFORMATION}
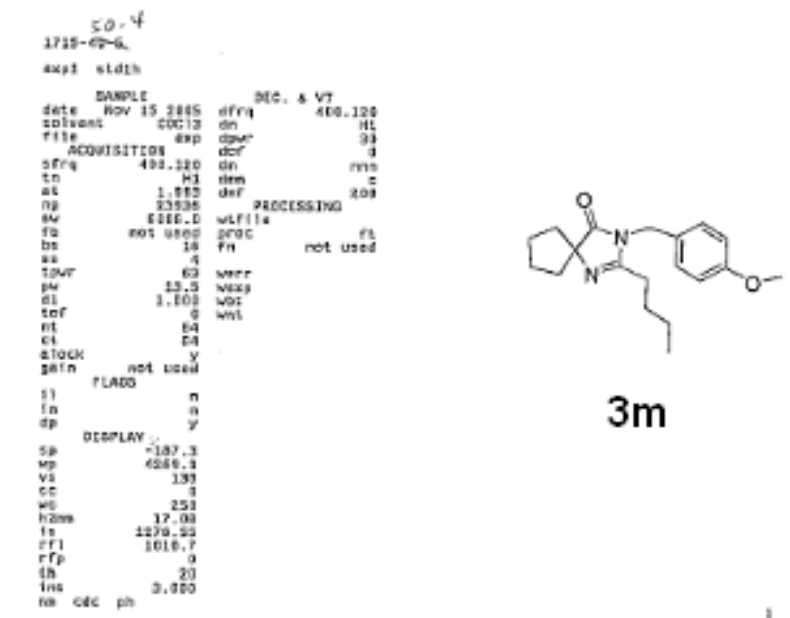

$3 m$

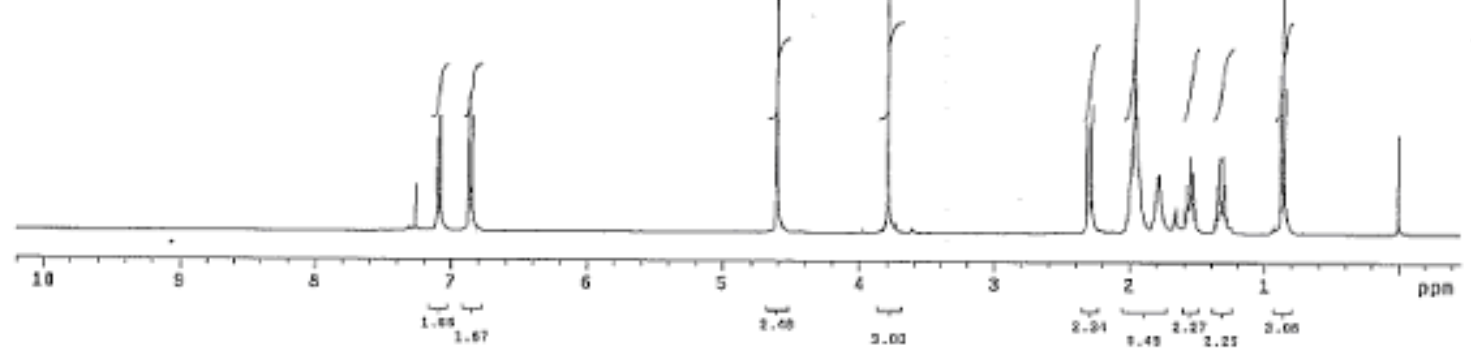


SUPPORTING INFORMATION

1715-60-4- 612

pules Sequanen: thpul

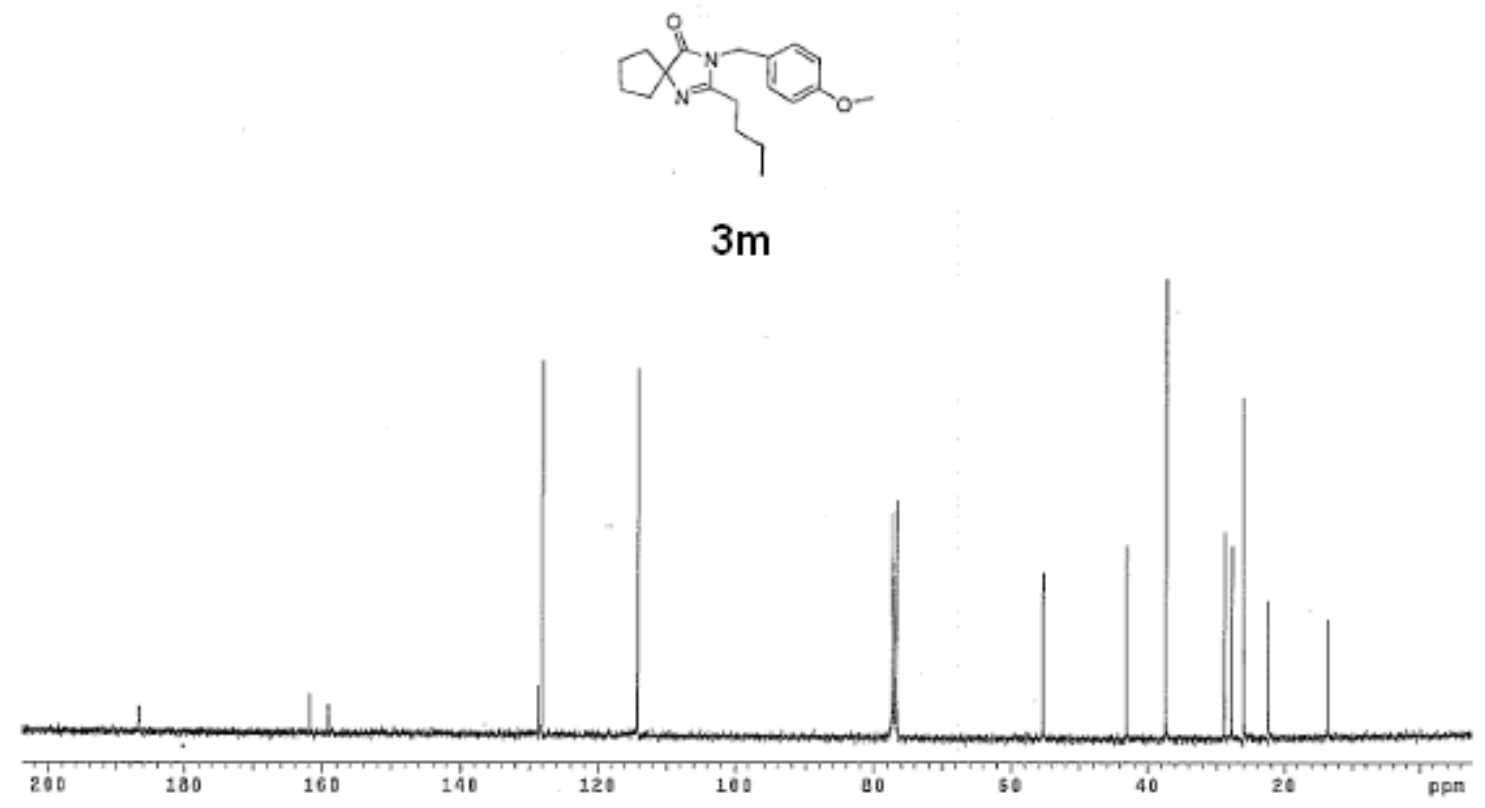




\section{SUPPORTING INFORMATION}

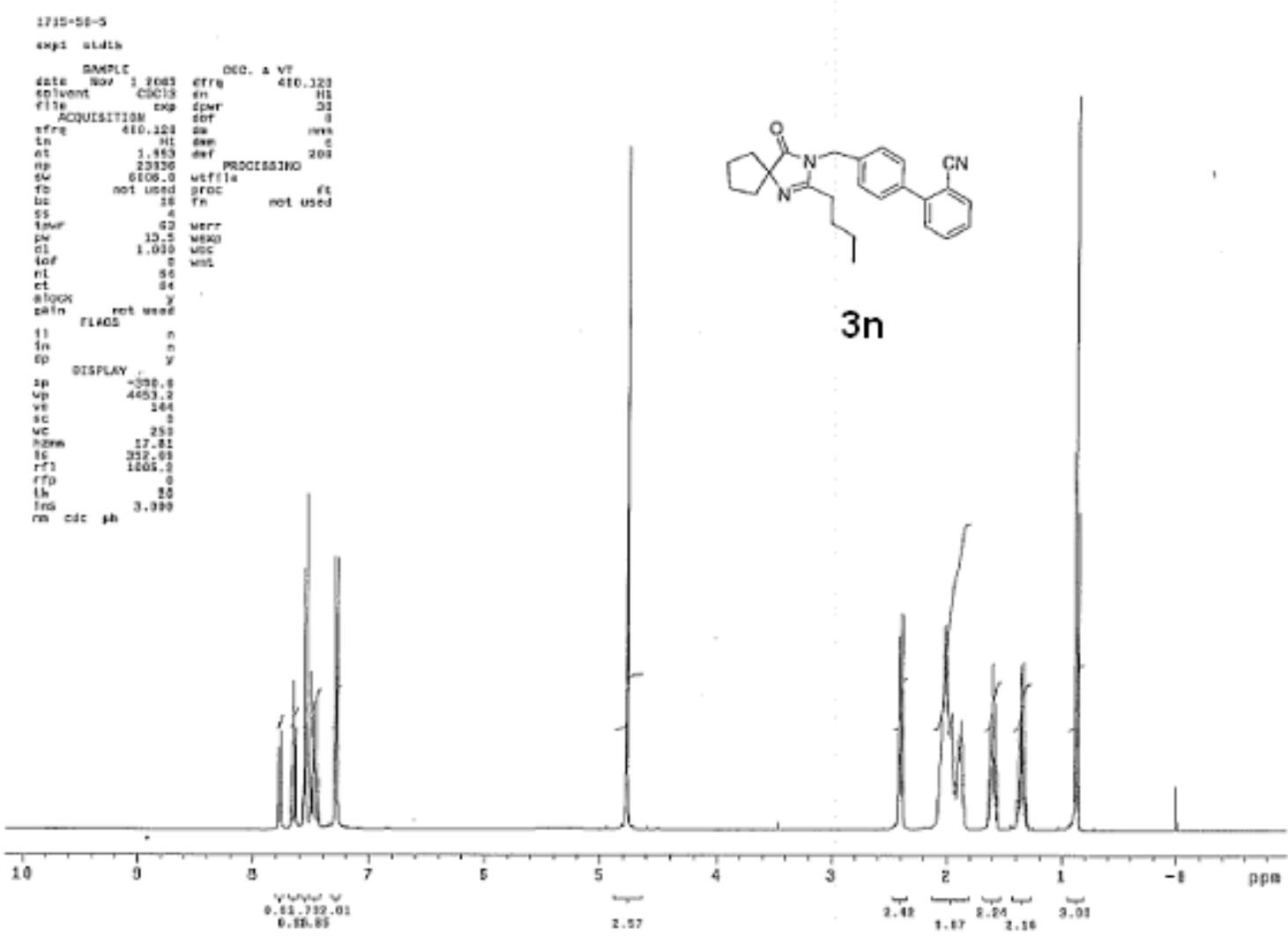


SUPPORTING INFORMATION

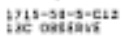

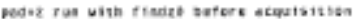

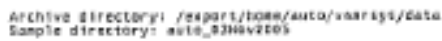

tulto soqsestent slpul

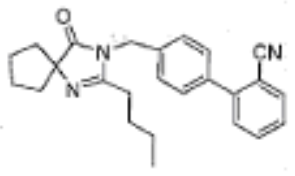

$3 n$

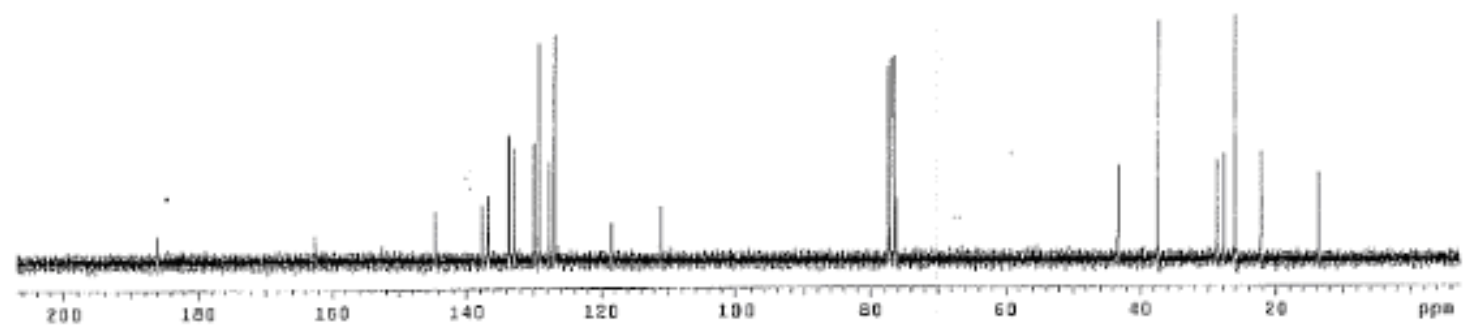




\section{SUPPORTING INFORMATION}

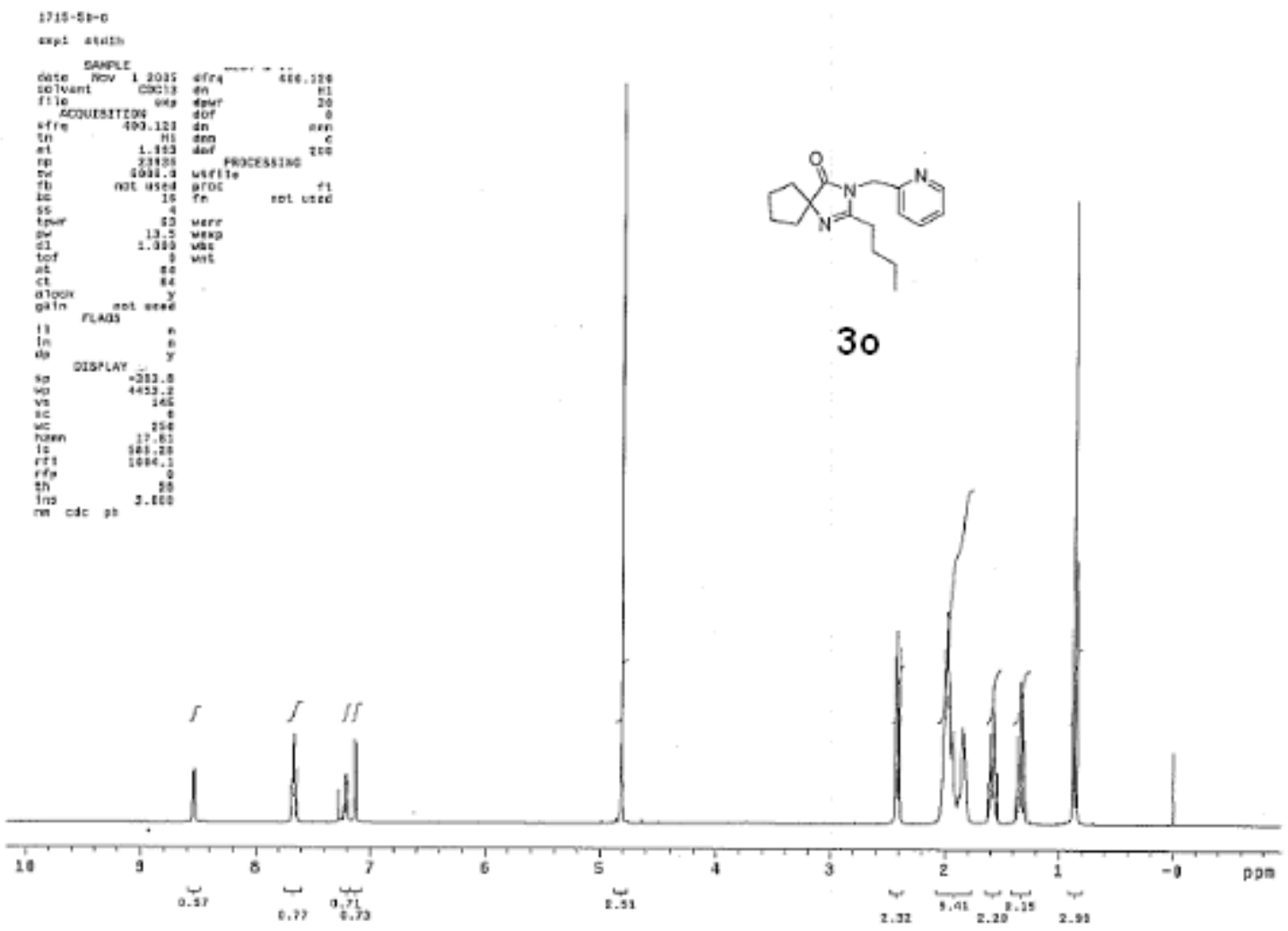




\section{SUPPORTING INFORMATION}
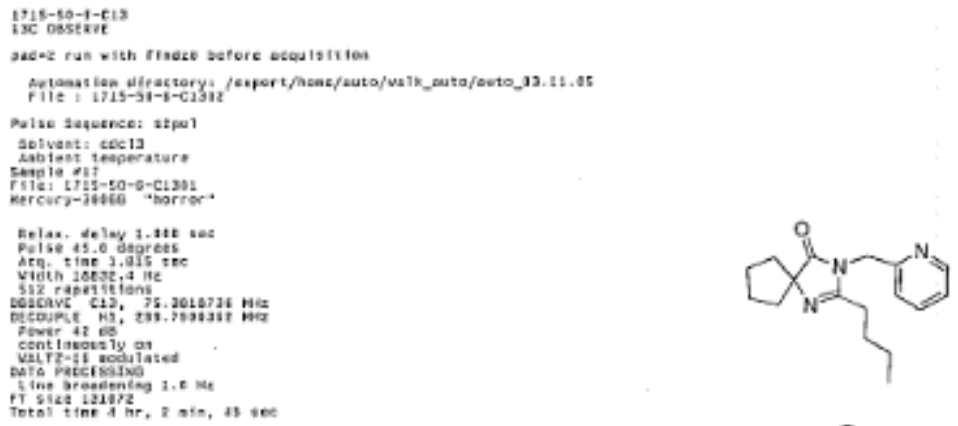

30

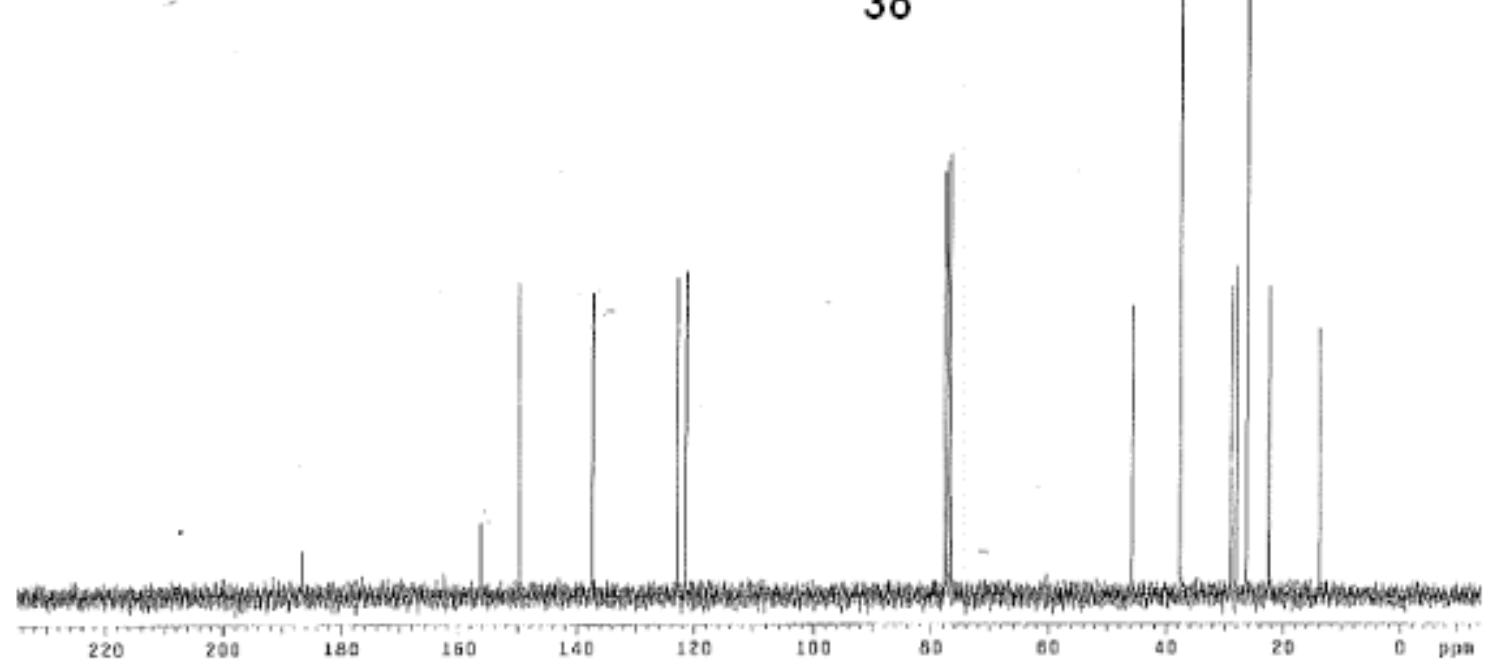




\section{SUPPORTING INFORMATION}
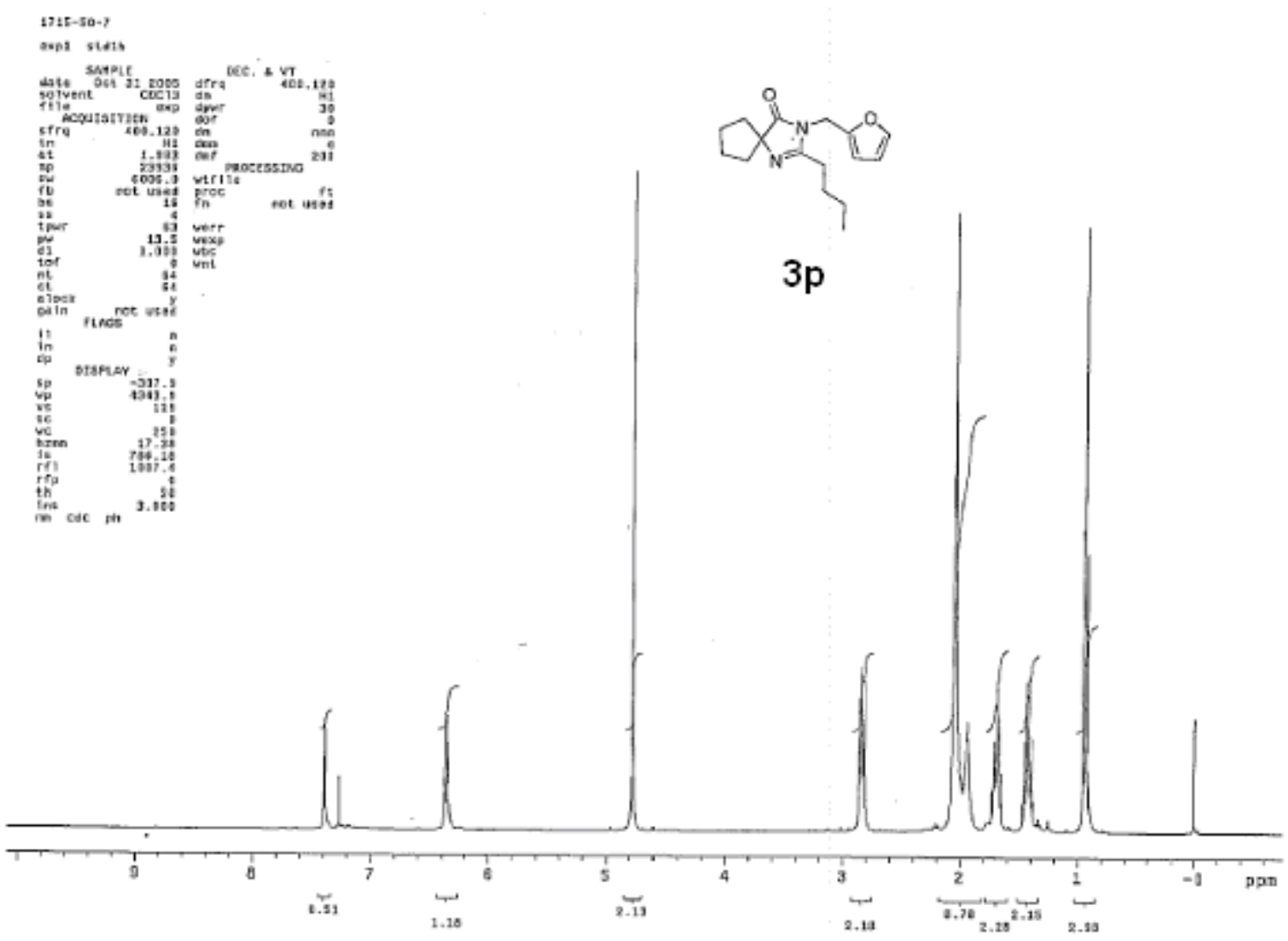
SUPPORTING INFORMATION
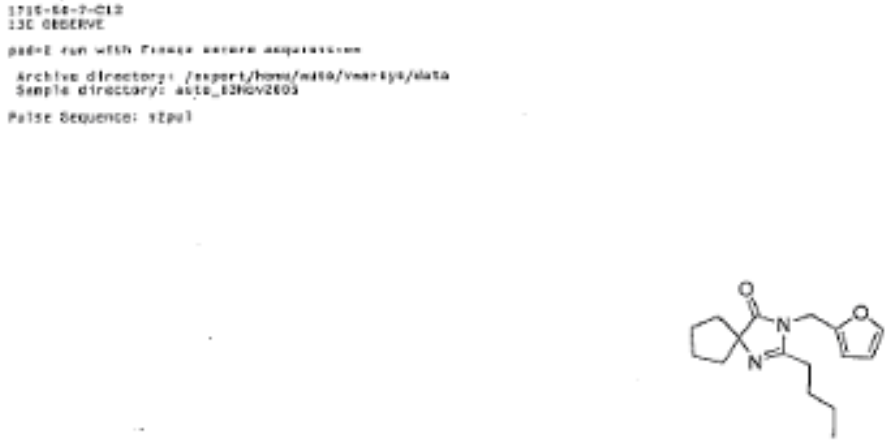

$3 p$

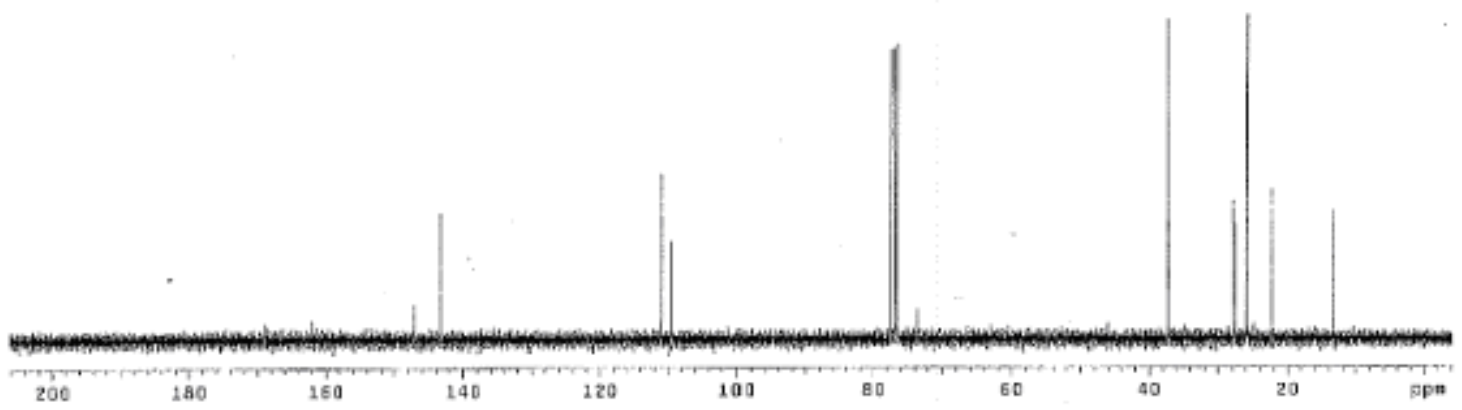




\section{SUPPORTING INFORMATION}

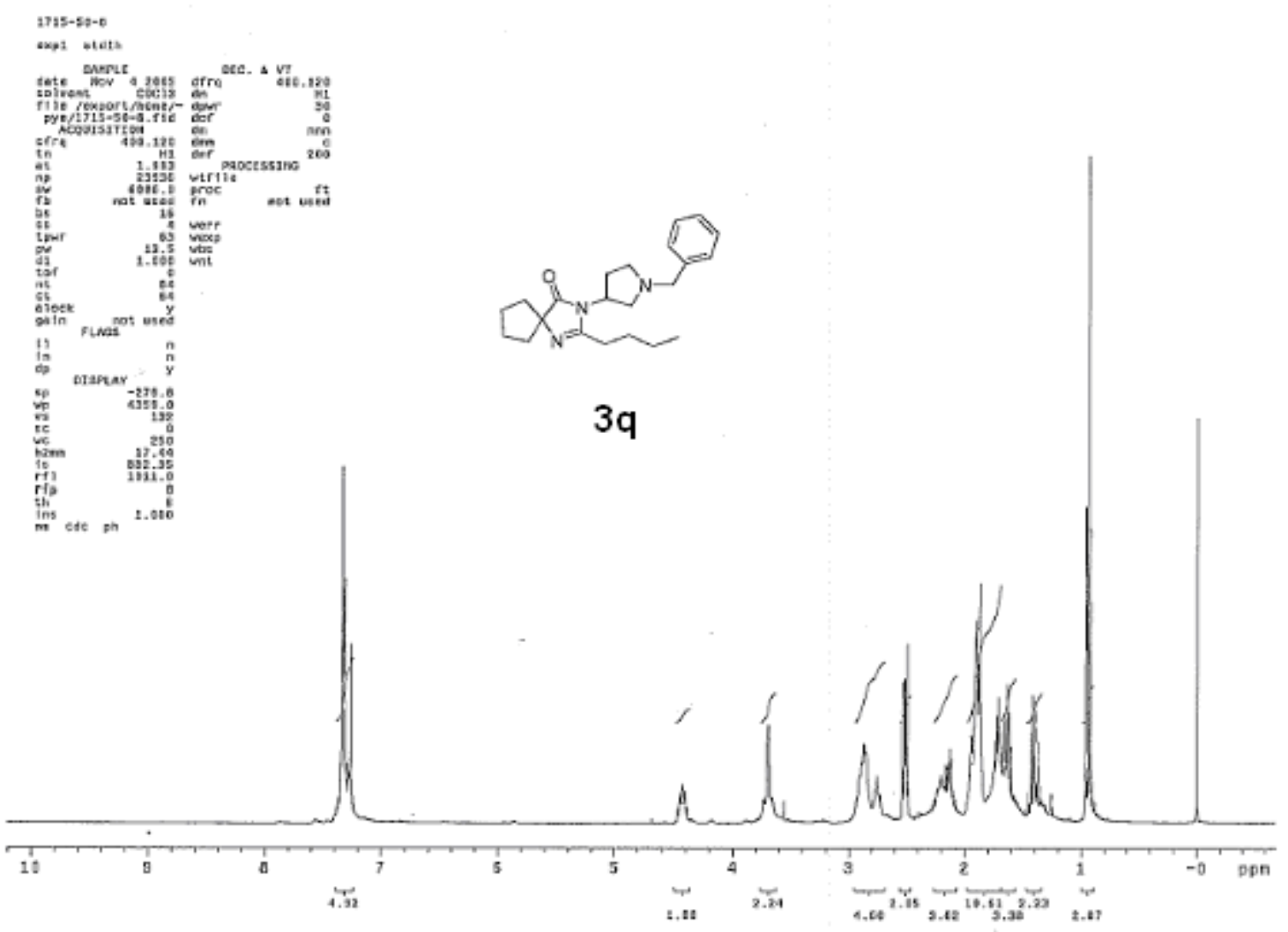


SUPPORTING INFORMATION

$1735-50-3-\leqslant 13$

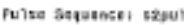

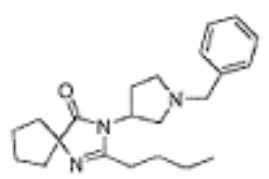

$3 q$

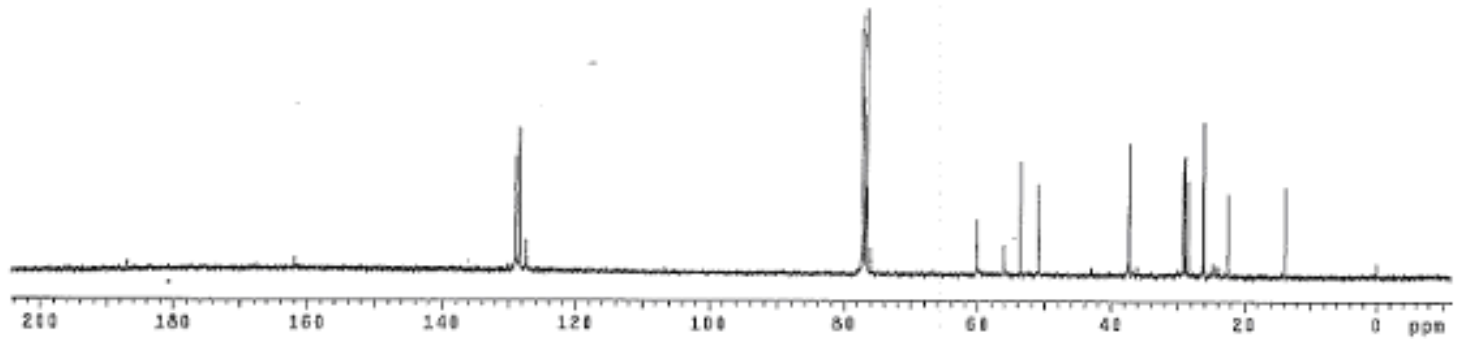




\section{SUPPORTING INFORMATION}
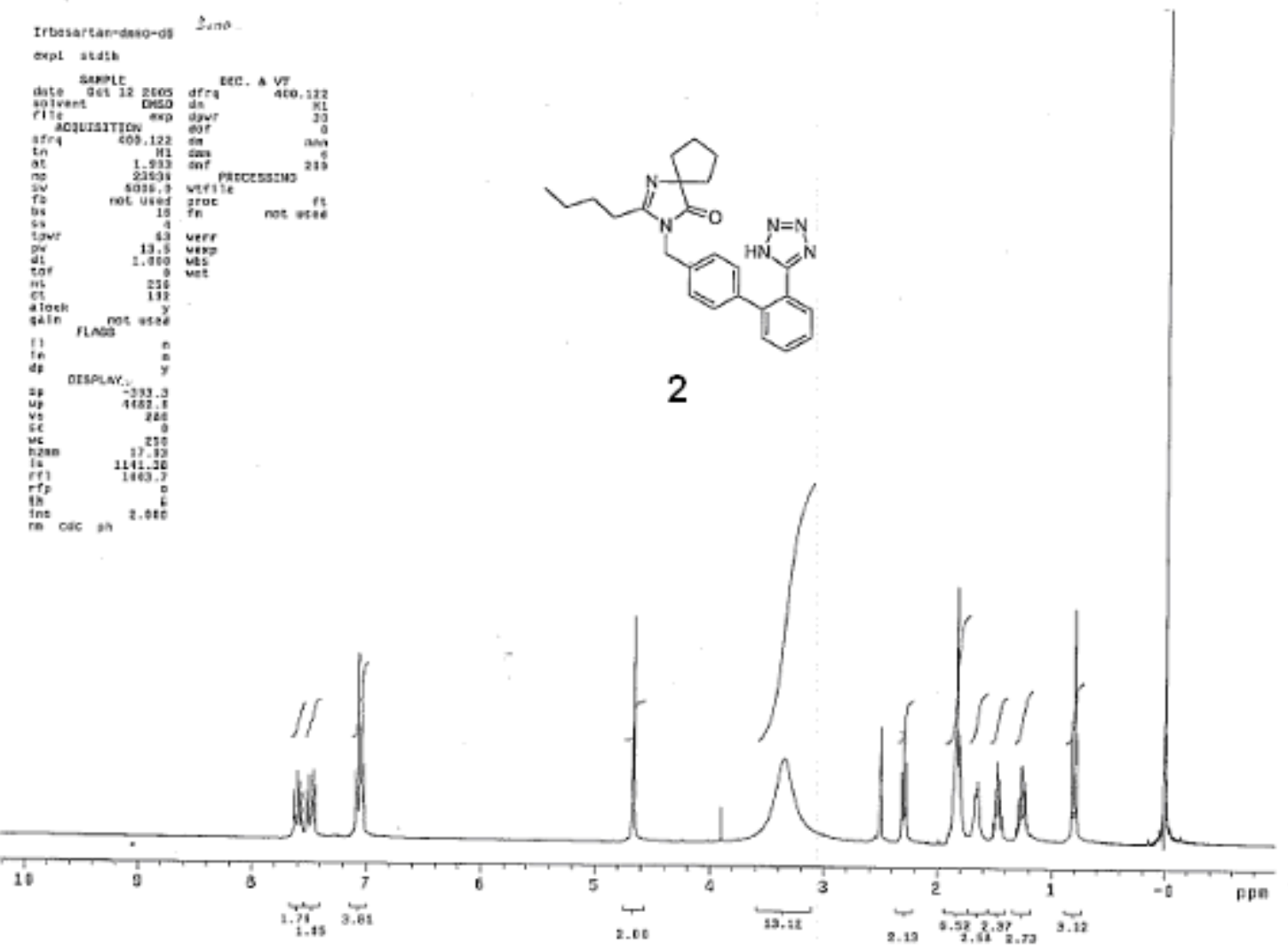
SUPPORTING INFORMATION
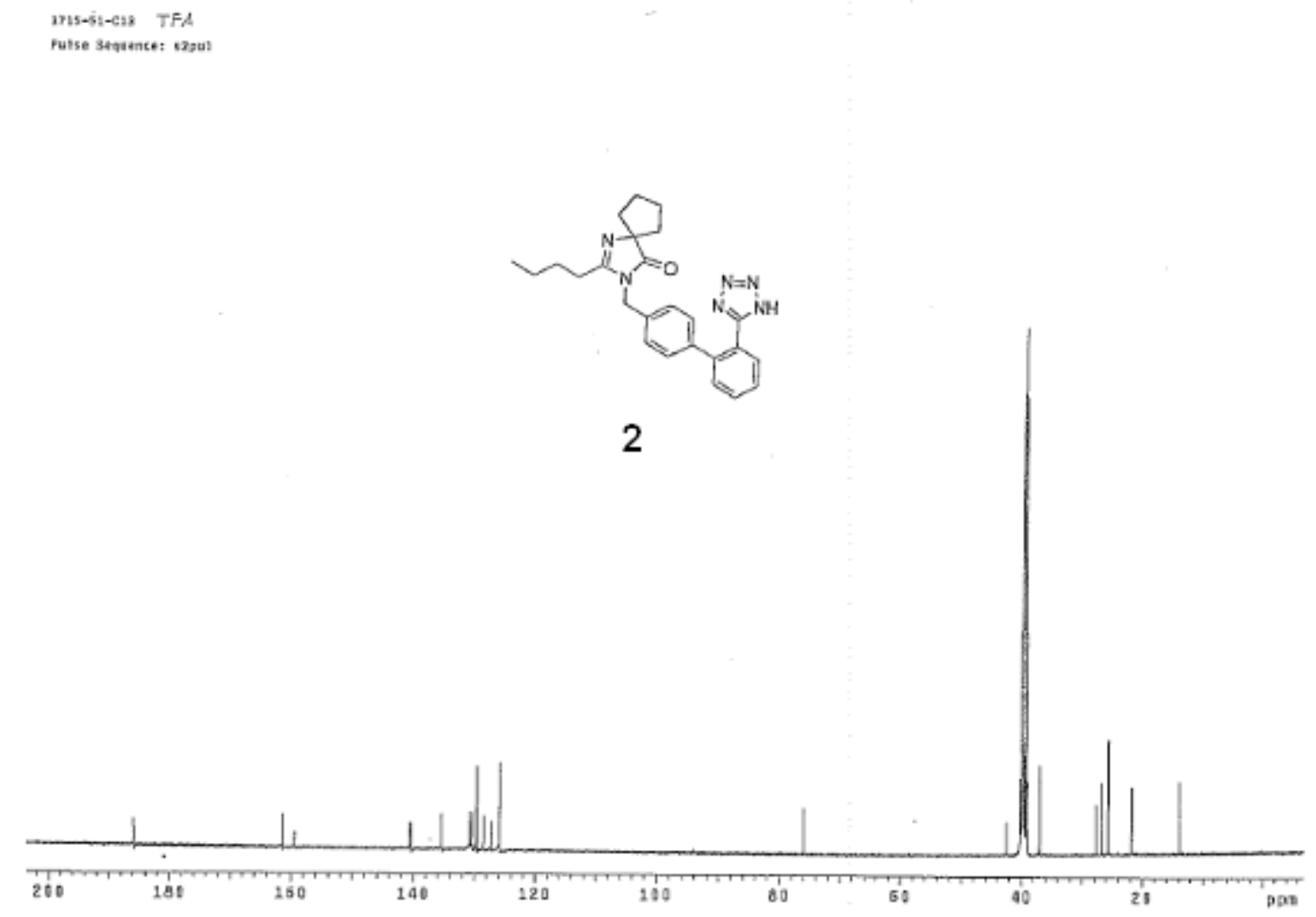